\title{
DIE MEDIASCHER MUNDART.
}

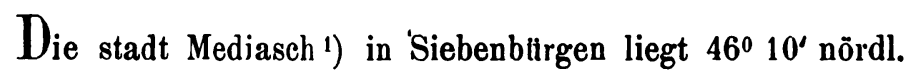
br. und $24^{\circ} 25^{\prime}$ östl. von Greenwich im tal der 'grossen Kokel', einem von 0 . nach w. fliessenden nebenfluss des Maros, in einer höhe von 937 fuss über dem adriatischen meer.

Die entstehungszeit der deutschen colonie von Mediasch lässt sich nicht mit sicherheit bestimmen. Die frage, ob die grundung des ortes in die zeit der ersten grossen einwanderung von Deutschen unter könig Geisa II. (1141-61), oder erst in das 13. $\mathrm{jh}$. fältt, ist unentschieden und historische anhaltspunkte zu ihrer lösung kaum vorhanden. Die erste urkundliche erwähnung der villa Medjes stammt aus dem jahr 1283. Der ort war der mittelpunkt und seit dem ende des 15. jh. auch der vorort eines besondern sächsischen gaues, der den namen duae sedes 'die zwei stuhle' (Mediasch und Schelk) fuhrt, zum unterschied von den 'sieben stuhlen', d. i. der fruheren provincia Cibiniensis. Unter könig Wladislaus II. (1490-1516) wurde der markt (oppidum) Mediasch zur stadt erhoben.

Nach der letzten officiellen volkszählung (1880) hat die stadt 6469 einwohner, ohne militär; darunter sind Deutsche 3470, Rumänen 1999; Magyaren 719, Zigeuner 188, ohne sprache (kinder) 182.2)

Was die beschäftigung der einwohner betrifft, so kann ich - da die bisher erschienenen publicationen des statistischen

1) Der name Mediasch schliesst sich an das ungarische Medgyes an, die daneben gebräuchliche benennung Medwisch an das mundartliche $m e^{2} d v o s$.

2) Die geographischen und historischen daten verdanke ich der guite des herrn gymn.-prof. Werner aus Mediasch; die statistischen zahlen sind die vom statistischen bureau in Budapest publicierten. 
bureaus sich noch nicht auf die berufsstatistik erstrecken nur aus eigener kenntnis der verhältnisse hinzufugen, dass die hauptbeschäftigung der bevölkerung in wein- und feldbau besteht; unbeteiligt an der landwirtschaft sind - ausser den besitzlosen Zigeunern - nur etwa beamte, lehrer und wenige kaufleute (kleinhändler). - An höheren lehranstalten befinden sich in Mediasch ein gymnasium, ein volksschullehrerseminar und eine ackerbauschule, die beiden ersten confessionell evangelisch A. B. - und deutsch, die ackerbauschule auch deutsch und von der 'sächsischen nationsuniversität' begrlundet und unterstützt.

Die deutsche bevölkerung spricht durchaus den heimischen dialekt; die deutsche schriftsprache wird in kirche und schule, eventuell, wenn nicht die magyarische staatssprache vorgeschrieben ist, im amt, ausserdem noch allenfalls im verkehr mit andern nationalitäten gesprochen; jedoch wird Magyaren und besonders Rumãnen gegentiber fast durchaus deren eigene sprache gebraucht. Da taglöhner und diensthoten meist rumänischer nation sind, spricht der grösste teil der Deutschen von kind auf auch den hier gebräuchlichen rumänischen dialekt.

Die sprache des weitaus grössten teils der deutschen bevölkerung ist noch sehr wenig vom schriftdeutschen beeinflusst; wo einflusse stattgefunden haben, sind sie meist auf den ersten blick als solche zu erkennen. Grössere einwirkungen hat naturlich die sprache der gebildeten erlitten; doch beziehen sich dieselben zumeist auf die syntax, weniger auf die flexion.

An die sprache des breiten teils der bevölkerung habe ich mich in der folgenden darstellung gehalten und in zweifelhaften fällen mich nicht gescheut, durch vergleichung mit den mundarten der umliegenden dörfer die entscheidung zu treffen.

$\mathrm{Ob}$ fremde sprachen, besonders rumänisch und magyarisch (szeklerisch) den dialekt beeinflusst - darüber enthalte ich wich noch jeden urteils. Soviel glaube ich jedoch sagen zu durfen, dass wenn beeinflussungen des organismus der sprache stattgefunden haben, diese nur in veränderungen der 'indifferenzlage' oder des accents bestehen können, und dies darum, weil lautstand und flexionsweise - besonders des magyarischen doch zu weit verschieden sind von denen des deutschen, als dass in diesen punkten einwirkungen hätten statthaben können. 
Nicht hierher zu rechnen ist also die tatsache, dass von eigentlichen bauern, weil sie eben im landwirtschaftlichen verkehr so häufig die rumänische sprache reden, oft rumänische benennungen, besonders fü: landwirtschaftliche dinge gebrauctit werden. Dies ist mir allerdings mehr aus der umgebung, als aus Mediasch selbst bekannt.

\section{LAUTLEHRE.}

\section{A. Die laute der mundart phonetisch betrachtet.}

I. Einiges uber den allgemeinen charakter der mundart.

\section{Indifferenzlage.")}

§1. Die indifferenzlage - ich kann freilich nur sagen meiner sprachorgane - ist beim aufrechtstehen oder sitzen im wesentlichen folgende: die lippen sind geschlossen, die kiefer stehen soweit von einander ab, dass die ränder der ober- und unter-schneidezähne etwa in derselben horizontalebene liegen. Der untere saum der zungenspitze ruht auf der schneide der unteren schneidezähne, mit ihrem rutcken stemmt sich die spitze merklich gegen die oberen schneidezăhne und die vorderen alveolen. Einen zweiten, ebenso fublbaren druck ubt die zunge aus ungefähr auf die grenze des harten und weichen gaumens. Sie fullt den ganzen ihr freien raum der mundhöhle aus. - Am wichtigsten jedoch zur charakterisierung der indifferenzlage ist die (im vergleich zum buhnendeutschen) sehr energische senkung des kehlkopfes, wodurch besonders alle sonorlaute ein gutturales timbre bekommen.

$A \mathbf{n m}$. Durch die stellung des kehlkopfes erbălt die ganze mundart ein gepräge, das sie in directen gegensatz stellt zu dem bühnengemässen sog. 'vorne sprechen'. Am merklichsten tritt dies bei vielen individuen in pathetischer rede hervor, sehr hänfig habe ich es bei kanzelrednern gehört.

\section{Accentverhältnisse.}

Diese schwierigen und fur das ganze einer sprache so charakteristischen verhältnisse glaube ich am besten klarzu-

1) Vgl. Sievers, Phonetik ${ }^{3}$ s. 20. 
legen, wenn ich einige, durch sie zum mindesten mitbewirkte erscheinungen in der mundart beschreibe.

a) Silbenbildung und -trennung.

§ 2. Der dialekt liebt offene silben; geschlossene silben kommen nur in wörtern vor, in denen auf den sonanten (Sievers, Phon. ${ }^{3} 39$ f.) einer silbe - sei er kurz oder lang consonantengruppen folgen, deren erstes element schallkräftiger ist als deren zweites; in diesem fall wird das erste element zur schliessung der ersten, das zweite zum anheben der zweiten silbe verwendet. - Stets geschlossen sind diejenigen silben, in welchen auf den sonanten ein $\dot{n}$ oder $x$ folgt, was sich aus der geschichte dieser laute erklären wird. Die silbengrenze fällt hier stets in diese beiden laute, wenn auf dieselben der sonant der nächsten silbe folgt.

§ 3. Die nämlichen principien der silbentrennung, wie im einzelnen wort, widerholen sich auch in der zusammensetzung und im zusammenhang der rede, wobei das einzelne wort sich ganz den sprechtakten unterordnet. Beispiele hierfur werden sich in den folgenden $\S \S$ dieses abschnittes finden.

b) Behandlung des aus- und anlauts (in der composition und) im zusammenhang der rede.

$\S$ 4. Alle im wortauslaut stehenden stimmlosen laute, welchen ursprungs immer, werden vor anlautendem vocal, wenn ihnen nicht ein anderer stimmloser laut vorangeht, stimmhaft.

A nm. Es werden also nicht nur die auslautend aus sonst stimmhaften lauten verstummten, sondern auch diejenigen stimmlosen laute im angegebenen fall stimmhaft, welche sonst im wortinnern stumm bleiben.

Beispiele: $a^{2} s$ ! $i^{2} \partial-s n$ (iss! essen) - aber: $a^{2}-z u^{2} n-d r a^{2} x k^{2}$ ! (iss und trink!); šle $e^{2} f$, šl $e^{2} f-n$ (schlief, schliefen) - aber:

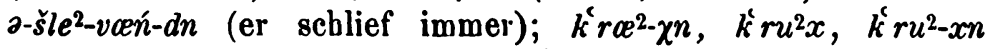
(kriechen, kroch, krochen), aber: $k^{i} r e^{2}-j a e^{2}-n a n !$ (kriech hinein!); a-k $r u^{2}-z^{2}-r \propto o s$ (er kroch heraus); dagegen: $m a^{2}-t^{t} \hat{\imath} f-t^{\dagger} u^{2}-x \hat{o r}$ (mit haupt und haar) $=m e^{2} t^{\dagger}$, hîf $\hat{t}, u^{2} x$, hôr .

$\S 5$. Alle anlautenden stimmhaften zungengaumenlaute (ausser $l, r, n, \dot{n}, x)$ und lippenlaute (ausser $m$ ) werden nach auslautendem stimmlosen laut - gleichviel, ob dieser ursprüng. 
lich stimmlosoder nur als im auslaut stehend stimmlos geworden - auch stimmlos, und zwar zur stimmlosen lenis. Von $l, r$, $m, n[n, n]$ verstummt mindestens der eingang; z. b. va-sr; a-gacat va-sr (ein gut wasser), bân; an-hîš-bam (ein ht:bscher baum),

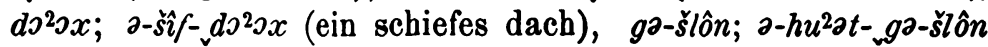
(er hat geschlagen), zôn; va-t zôs $t^{t} \partial$ ? (was sagst du?), jố-jr; $a-l i^{2} \chi-t, j \hat{a}-j r$ (ein schlechter jäger).

§6. Im zusammenhang der rede verliert jeder anlautende vocal - ausser, wenn eine pause vorangieng - den kehlkopfverschluss; dies ist selbst bei vorausgehendem vocalischen auslaut der fall, z. b. vô- $\partial^{2} \supset s-\hat{t} r$ ? (wo asst ihr?), $d \hat{\imath}-\hat{\imath} n-t^{2} i^{2} \chi($ der - in der zeit - vorige), tsvô-ô-ma-zn (zwei ameisen).

A $\mathbf{n} \mathbf{m}$. Zu den beiden letzten beispielen vgl. Sievers, Phon. ${ }^{3} 180 \mathrm{f}$. $188 \mathrm{f}$. über drucksilben und -grenzen.

$\S 7$. Begegnen sich im aus- und anlaut zweier wörter verschlusslaute, so explodiert der erstere von ihnen erst im zweiten.

A n m. Eine ausnahme macht nur vorangehendes $k$, welches eigentlich affricuta ist.

Begegnen sich in derselben stellung die gleichen tenues, so erscheint für beide nur die eine; auch $k^{k} k^{k}$ verschmelzen hier in ein $k$. - Treffen tenuis und media des gleichen lautes zusammen - die media nimmt dabei, weil nie eine solche auslauten kann, stets die zweite stelle ein - so resultiert die stimmlose lenis desselben lautes; - z. b.: $h 0^{2} t^{t}, b 0^{2} 2 n t^{c}-$

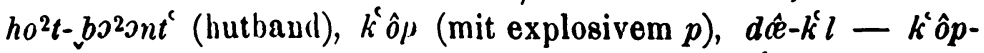
$d \hat{e}-k^{k} l$ (wassergefïssdeckel), dagegen $t s i^{2} k^{k}-t s a k^{c}$, nicht $t s i^{2} k$ tsak', baḱ-trôx, nicht bak-trôx (backtrog), gaeat, $t o^{2} \partial^{2}-z r-$ a-gaca-t $0^{2} \partial^{2}-2 r$ (eine gute tasche), bo $20 t, d i^{2} \chi-\chi-b \partial^{2} \partial-d i^{2} \chi$ (ich bat dich), tsôp, ban-dl-tsô-ban-dl (zopfband), gla $\boldsymbol{c}^{2} \hat{k}$, $k^{2} i^{2} \partial-j l n-m c^{2} t-g l c^{2}-k^{2} i^{2} \partial-j l n$ (mit glick kegeln).

§ 8. 'Treffen sich aus- und anlautend dieselben dauerlaute, ausser den unter $\S 6$ behandelten vocalen, so verschmelzen sie zu einem einzigen; aus $f-v, s-z, \chi-j$ wird dabei $v, z, w$; -

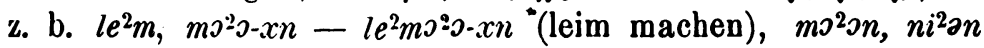

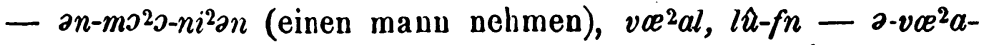
lu-fn (er will lanfen), $v u^{2} a r, r e^{2}-n a n-v u^{2} \partial-r e^{2} n-t s t a ?$ (wohin rennst du?), af, $v u^{2} \partial r-d n-a-v u^{2} a r-d n$ (auf warten), $a^{2} s, z a e^{2} a l-$ 


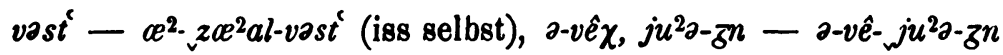
(wegjagen).

§ 9. Alle auslautenden flexions- $n$, ob in fruberen sprachstănden gedeckt oder ungedcckt, aus fruherem $n$ oder $m$ hervorgegangen, schwinden im zusammenhang der rede, wenn das năchste wort nicht mit vocal, $h$ oder $d, t(t s)$ anlautet. An stelle des geschwundenen $n$ erscheint, wenn ihm ein nichtvocal vorangieng, $\partial ;-$ z. b. goea-dn-nô-vnt (guten abend),

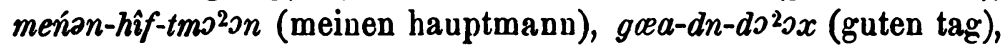
deñan-tsyu-rn (deinen zorn), aber: $d \partial-g a x a-d \partial-b r \hat{o}-d \partial-m \partial^{2} \partial-x n$ (den guten braten machen).

A n m. In einigen (isolierten) wörtern unterliegt demselben gesetz auch stammhaftes $n$, z. b. $\check{s} y u(n)$ schon, $u^{2}(n)$ an, $a^{2}(n)$ in, $f u^{2}(n)$ von, $\hat{\imath}(n)$ ein, masc. und neutr., aber fem. $\hat{i} n$.

In meń, deń, zeń - mein, dein, sein - und zeń [oder $z e^{2} n$ ] esse wird $n$, wenn es in den auslaut tritt, ebenso behandelt, wie die obigen $n$; nur im femininum fällt es nie ab; z. b. $m e^{2}-m v^{2} o n$ (mein mann),

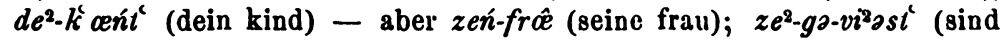
gewesen).

$\S 10$. Tritt eine gruppe cons. $+l, r$, nasal $(l, r$, nasal in sonantischer function) in den auslaut, so wird, wenn das folgende wort mit einem vocal beginnt, der sonant $(l, r$, nasal) in der weise geminiert, dass der erste teil als sonant weiter fungiert, der zweite teil aber als consonant vor den folgenden vocal antritt. Dies ist meines wissens der einzige fall wirklicher geminata in der mundart. Zwischen l-r schiebt sich jedoch gerne ein $a$ ein, wodurch die gemination verhindert wird; z. b. $v e^{2}-f l, o^{2} \jmath^{2} r-v e^{2} f l-l o^{2} \jmath$ r (wieviel eier?), for-tsce $2-r l$, $a n^{\prime}-d n-n e^{2}-t f a r-t s a^{2}-r l-l a n^{\prime}-d n$ (verzettele nicht immerl), $v e^{2} d r$,

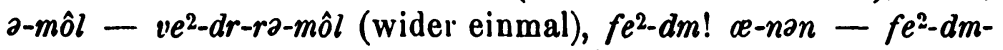
$m e^{2}-n a n !$ (fädeme ein!), zi $i^{2}-v n, \hat{e}-v n t^{c}-z i^{2}-v n-n \hat{e}-v n t^{c}$ (sieben abende); dagegen: $k^{k} a^{2} a-l r, u^{2} \partial-v n-a^{2} n-d \partial-k^{k} a c^{2} a-l \partial-r u^{2} \partial-v n$ (in den keller hinab).

c) Behandlung der vocale in nicht-haupttonigen silben.

§ 11. Sämmtliche vocale der nicht-haupttonigen silben sind zu a geworden oder ganz geschwunden; letzteres ist der fall in der nähe von $l, r$ und nasal, ferner im auslaut, so dass der dialekt keinen einzigen nicht zur stammsilbe gehörigen vocal im wortauslaut hat, mit ausnahme des $\partial$, welches nach abfall von $n$ in zusammenhang der rede auslautet, 
vgl. § 9. - Vocalschwund in der nähe von $l, r$, nasal hat jedoch nicht statt zwischen combinationen von $m, n, \dot{n}, x$; ausserdem ist noch binzuzufugen, dass in gedehnter, 'singender' rede in allen fällen ein a widergestellt werden kann, sehr leicht besonders zwischen $l-r$, weniger schon zwischen $r-l$.

Da die sache so einfach daliegt und im folgenden sich beispiele genug finden werden, brauchen hier wol nicht besondere angeführt $\mathrm{zu}$ werden.

\section{Das lautsystem der mundart.}

\section{Die rocale.}

§ 12. Bei der transscription der vocale habe ich mich so eng wie mōglich an die von Sievers in seiner 'Phonetik' 3. auf. p. 95 angenommene Bell'sche vocaltabelle gehalten. $\mathrm{Zu}$ berücksichtigen ist dabei stets, was $\S 1$ uber die indifferenzlage, besonders die stellung des kehlkopfes gesagt ist.

a) Gutturale vocale: $a \ldots u, u^{2} ; 0, o^{2} ; 0,0^{2}$.

b) Palatale vocale: $i, i^{2} ; e, e^{2} ; \propto, \varkappa^{2} ; y \ldots \propto, \varkappa^{2}$.

c) Ein laut, welcher entsteht, indem durch das am geringsten aus der indifferenzlage gerückte ansatzrohr ein exspirationsstrom geleitet wird: a - Sievers' a (voice glide) a. a. 0.173 .

d) Stimmloser vocal: $h$, '-

Anm. 1. Durch den exponenten 2 drïcke ich die 'offenheit' des vocals aus; den 'geschlossenen' vocal lasse ich ohne nähere bezeichnung.

Anm. 2. Das zeichen $h$ gebrauche ich - um vom allg. schriftgebrauch nicht zu sehr abzuweichen - im wortanlaut [und zwischen vocalen], den asper dagegen bei den 'aspiraten'.

Ann. 3. Die rucale a) - c) drilcken anlautend auch den vorangehenden kehlkopfverschlusslaut mit aus, wenn derselbe nicht nach $\S 6$ weggefallen ist.

Anm. 4. An zeichen filr diphthonge habe ich verwendet:

a) für ächte diphthonge: $e^{2} e, \partial^{2}$.

b) fiir unächte diphthonge: $i^{2} a, y u, \propto x o, \alpha^{2} o^{2}, \propto x a, a^{2} a, o^{2} \partial^{2}, u^{2} \partial$. Die gerundeten vocale $y, a, a^{2}, 0, \partial^{2}$ kommen nur in diphthongen vor. - Die gesammtquantität von $a^{2} o^{2}$ und $e^{2} a$ ist geringer als die von $\propto o$ resp. cea.

Anm. 5. Ueber die unmöglichkeit einer ganz genauen transscription ist hier kein wort zu verlieren, ebensowenig luber die damit zusammeuhängende unmöglichkeit, eine phonetische transscription wider richtig zu lesen. Am fühlbarsten wird dies bei den vocalen. Be- 
deutend leichter sind schon die 'consonanten' zn beschreiben und zu lesen, und daranf vertrauend habe ich viele nicht gan7. unmerkliche vocalschattierungen direct unbezeichnet gelassen, weil ich glaubte, dass dieselben zugleich mit den sie umgebenden 'consonanten' indirect mitbezeichnet seien, sicher aber der hieraus entspringende mangel leichter wiegt, als der durch allzuviele vocalzeichen entstehende fehler der unübersichtlichkeit.

\section{Die liquiden.}

$\S 13$. Alle $l$-laute sind mit demselben zeichen $l$ transscribiert worden, weil sich alle arten in geringen abständen um eine stellung der organe bewegen, die nachher $\mathrm{zu}$ beschreiben ist. Verschieden von den tibrigen $l$ ist dasjenige, welches vor $t s ̌$ steht: bei diesem articuliert nämlich der zungensaum an den hinteren alveolen; da sich dies aber schon aus der natur des $t$ yor $s$ ergibt, habe ich jenes $l$ von den obigen im zeichen nicht unterschieden. Bei diesen articuliert nicht der zungensaum, sondern der rücken der umgeknickten zungenspitze.

$\S 14$. Allen $l$ jedoch gemeinsam ist eine eigentumlichkeit, die wahrscheinlich mit dem object der streitfrage uber das gutturale $l$ verwant ist. Indem ich die $l$-laute als nächste verwante der vocale fasse, möchte ich mit hinweis auf die Bell-Sievers'sche vocaltabelle unser $l$ guttural-niedrig-geschlossen nennen, wenn ich das buhnendeutsche $l$ als palatal-hoch-offen auffassen darf. Genauer gesagt, die hintere masse der zunge wird energisch zurtickgezogen (guttural), wobei zugleich uber den ganzen zungenkörper sich eine spannung verbreitet (geschlossen); der mittlero teil der zunge senkt sich ganz beträchtlich (niedrig), wodurch wahrscheinlich der $u$-klang dieses $l$ hedingt wird. - Am leichtesten kann man den laut nachbilden, wenn man zuerst recht scharf $u^{1}$ articuliert, dann, durch aufheben der lippenrundung, zu $A^{1}$ gelangt und nun an der stellung der sprachorgane möglichst wenig ändernd die verbreiterte zungenspitze bis zu den alveolen hebt. Beim gewöhnlichen $l$, um dies nochmals zu erwähnen, articuliert nun der vorderste zungenrlucken an den alveolen, so dass der rand der zunge die schneide der unteren schneidezähne berluhrt; beim $l$ vor $t s ̌$ articuliert der zungensaum an den hinteren alveolen. 
$\S 15$. Die $r$-laute. - Diese alle habe ich durch das eine zeichen $r$ widergegeben, weil sie insgesamt arten, des gerollten zungenspitzen mittleren alveolar-r sind (Sievcrs p. 106). Alle andern $r$, welche nicht zungenspitz-r und gerollt sind, werden, wenn sie individuell vorkommen, als dialektwidrig empfunden.

A $\mathrm{nm}$. Von individuen, welche das gerollte zungenspitzen-r nicht sprechen können, sagt der volksmund spöttisch: za râtšn - welches letztere wort meinem gefühl nach um des anlantenden $r$ willen frei erfunden wurde.

$\S 16$. Von substitutionslauten für $r$ kommt - jedoch nur individuell - bei erwachsenen am häufigsten vor ein $\zeta$, etwa auf der grenze von hartem und weichem gaumen articuliert und stets mit einem kurzen vorgeschlagenen gleitlaut verbunden, welcher gleich ist dem laut, der durch explosion eines $g$ entsteht; diesem laut folgt $z$ hastig nach, wodurch der eindruck erzeugt wird, als schlage die zunge heftig gegen den gaumen an. Ohne zweifel will dadurch das rollen des zungenspitzen$r$ nachgeahmt werden.

Seltener ist das uvulare gerollte $r$.

Gehört habe ich einmal auch ein $v$ für $r$, ebenfalls mit dem kurzen vorgeschlagenen gleitlaut; dieses $v$ war jedoch niclit das gewöhnliche, wie z. b. in wasser, sondern die unterlippe zog sich etwas an den zähnen empor, so dass die oberzähne bereits an der feuchten schleimhaut articulierten. $b v \hat{a}-d v$ resp. bava-dav fur $b r \hat{\imath}-d r, v a-s v$ resp. $v a-s a v$ für $v a-s r$.

A n m. 1. Kinder lernen das $r$ meist erst mit dem 3., viele sogar erst mit dem 5., 6. jahr zungenspitz und gerollt sprechen; soweit meine erfahrung reicht, sprechen bis zil dieser zeit alle kinder das gew 8 hnliche $l$ fiir $r$, was für den schlagendsten beweis dafür anzusehen ist, dass in bewusstsein der mundart das nungenspitzen-r für das einzig berechtigte gilt.

A $n$ m. 2. Was die anzahl der zungenschläge beim 'rollen' betrifft, so mache ich bei selbst raschem sprechen zwei schläge; ich glanbe jedoch, dass der nur dialect sprechende mehr zungenschläge macht, besonders bei langsamem, singendem redevortrag.

\section{Die nasalen.}

$$
m, n, \dot{n}, x \text {. }
$$

$\S 17$. Das $m$ wird ähnlich gesprochen, wie das hd. $m$ auf der bühne, steht also in bezug auf seine dauer ungefähr in 
der mitte zwischen dem französ. $m$ und dem im hd. oft gehörten, fast zum gleitlaut verkürzten $m$.

$\S 18$. Alle $n$ fasse ich zusammen in dem zeichen $n$, womit ich einen nasal bezeichne, der mit dem vordersten zungenrlicken an den vorderen alveolen artikuliert wird, während die zungenspitze auf den unteren schneidezähnen ruhend mit dem oberen teil sich gegen die oberen schneidezähne stemmt.

$\S 19$. $\dot{n}$ bezeiclnet ungefähr denselben laut wie spanisch $\tilde{n}$, ital. $g n$, ungar. $n y$. Es wird genau ebenso artikuliert, wie $d^{\prime}$, uber welches $\S 23 \mathrm{zu}$ vergleichen ist.

$\S 20$. Mit $n$ bezeiclune ich sowol die vorderen, als auch die hinteren guttural-nasale; aus der natur des vorhergehenden vocals ergibt sich die unseres lautes von selbst.

\section{Die verschlusslaute.}

$\begin{array}{cccc}p & t & t^{\prime} & k \\ b & d & d^{\prime} & g \\ b & d & & g\end{array}$

§ 21. Die bezeichnungen der labialen verschlusslaute $p, b, b-$ sind ohne weiteres klar; $b$, ebenso auch $d$ und $g$ - stimmlose lenes - kommen nur in den $\$ \$ 5.7 .8$ besclriebenen fällen im zusammenhang der rede vor.

A nm. Ob $p$ gar nicht, oder nur sehr wenig aspiriert ist - oder wann das eine oder das andere - kann ich nicht entscheiden; vielleicht lässt sich jedoch daraus, duss es mir nicht widerstrebt, z. b. $p^{\prime} a-p^{\prime} a$ mit kräftiger aspiration zu sagen, schliessen, dass der laut leicht aspiriert sei. Oder ist dic aspiration bei $p$ univesentlich und nur folge des grösseren oder geringeren exspiratorischen nachdrucks, der auf eine silbe mit $p$ gelegt wird? Dieser ungewissheit wegen bezeichne ich die vielleicht vorhandene und wesentliche aspiration gar nirgends. - Wolff, Ueber den consonantismus des Siebenbürgisch-sïchsischen $34 \mathrm{ff}$. erwähnt keine aspirata $p$.

§ 22. Die dentalen verschlusslaute $t, d, d$ werden ganz so artikuliert, wie das $\S 18$ beschriebene $n$; eine ausnahme macht nur $t$ vor $\breve{s}$, welches fast cerebral artikuliert wird.

A $\mathrm{nm}$. $t$ ist anslautend, oder wenn ein vocal folgt, sehr stark aspiriert, fast affriciert, weniger, wenn es mit folgendem $r$ in den auslaut tritt. Folgen auf $t$ andere laute, als vocal oder $r$, so explodiert es erst in diesen, wodurch der charakter einer aspirata verloren geht; vgl. § 7. Häufig, bei singendem sprechen stets, ist $t$ auch bei folgendem $r$ in wortanlaut aspiriert: $t i^{2} \partial-d n$ und $t^{2} r i^{2} \partial d n$ (treten), aber immer $f u^{2} a-t^{i} r$ (vater). 
$\S 23$. Mit $t^{\prime}, d^{\prime}$ bezeichne ich die mouillierten formen von $t$, $d$. Man kann sie am leichtesten nachbilden, wenn man zuerst ein $i^{1}$ so geschlossen wie möglich artikuliert, dann, sonst die sprachwerkzeuge in derselben spannung behaltend, die zunge so an den gaumen legt, als wolle man $g$ resp. $k$ - die Sievers'schen $\zeta^{1}$ und $c^{1}$ - bilden, gleichzeitig aber auch weiter vorne die zunge für $d$ resp. $t$ einstellt und erst wenn der druck auf dem ganzen gebiet zwischen den artikulationsstellen des $g-k$ und des $d-t$ gleichstark verteilt ist, die explosion eintreten lässt.

A n m. $t^{\prime}$ und $d^{\prime}$ kommen nur mit folgendem $t$ und $d$ eng verbunden vor: $t^{\prime} t, d^{\prime} d$. - Ohne folgendes $t, d$ nur vor $\chi, j$ als 'unorganische' einschiebungen.

$\S 24$. Mit $k, g, v$ habe ich alle vorderen und hinteren gutturale zusammengefasst.

Anm. $k$ erscheint stets affriciert. Dass das zweite element eine spirans und kein blosser hauch ist, geht daraus hervor, dass $k$ nie im folgenden laut (richtiger buchstaben) explodiert und jenen anbängenden zweiten laut stets behält. Vgl. \$ 7. Ich schreibe jedoch nicht $k x$, sondern $k$, weil wir gewohnt sind, $k, t$ als aspiraten zu fassen.

§ 25. Zu den erwähnten verschlusslauten ist noch hinzuzufligen der kehlkopfverschlusslaut, uber welchen vgl. $\$ 6$ und $\S 12$ a. 3 .

\section{Die spiranten.}

$\begin{array}{ccccc}f & s & \xi & \chi & x \\ v & z & \check{z} & j & z \\ v & z & & j & \end{array}$

§ 26. Die zeichen $f, v, v$ sind leicht verständlich, ebenso $x, \xi$, in denen ich alle ach-laute, und $\chi, j, j$, in denen ich alle ich-laute zusammenfasse.

$\S 27$. Wenn ich $s$ oder $z$ artikuliere und darauf den mund öffne, füllt die zunge den raum zwischen den zähnen des unterkiefers vollkommen aus und zwar so, dass der scharf ausgeprägte zungensaum gerade die schärfe der schneidezähne und nach hinten die innere kante der backenzähne berlihrt. Vie mittellinie des zungenrüekens senkt sich ein wenig ein. Schliesse ich nun wider den mund und artikuliere abermals $s, z(z)$, so erhebt sich aus der eben beschriebenen lage zu beiden seiten der mittellinie der vorderste teil der zunge, eine 
rinne bildend, zu den alveolen; das reibegeräusch entsteht an den oberzähnen.

$\S 28$. Zu $\check{s}$ und $\check{z}$ gelangt man am leichtesten, wenn mau recht kräftig $u^{1}$ artikuliert und, die rundung der lippen erweiternd, die zunge in ganz derselben spannung zum harten gaumen erhebt.

Anm. 1. $\check{z}$ entsteht regelmässig aus $\check{s}$, wenn dieses auslautend vor anlautenden vocal zu stehen kommt, sonst nur vereinzelt.

Anm. 2. Zu $\check{z}$ und $z$ gibt es in unserer mundart keine entsprechenden stimmlosen lenes, weil $\check{z}$ und $\zeta$ nic anlautend vorkommen und stimmlose lenes nur in anlaut nach vorhergehendem stimmlosem auslaut zu finden sind, vgl. $\$$ 5. 7.8.

\section{B. Die laute der mundart historisch betrachtet.}

I. Die westgermanischen stammvocale.

\section{a) Kurze vocale.}

1. Westgerm. $\check{a}$.

$\S 29$. Altes $\breve{a}$ wird zu $\jmath^{2}$ vor gruppen von $l, m, n+$ consonant und vor $m m$; ferner vor altem $k>$ (im dialekt geworden zu) $x$. Umlaut: vor $l+$ cons.: $a^{2} a$; vor $m+$ cons. und $m m: e^{2}$; vor $n p, n d: o(n d)$; vor $n t: i^{2}$; vor $k>x$ : $\hat{e}$. Beispiele:

1. Vor $l$ + cons.: gavoºlt (giwalt), ho²oldn (haltan),

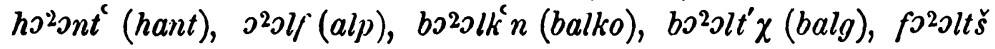

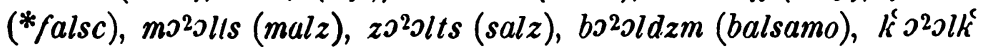
(chalch). Umlaut: $\hat{\imath} j^{\prime} x^{2} a l d i^{2} \chi$ (einfaltîg), $a^{2} u l d r$ (eltiron).

A $\mathrm{n} m$. Vereinzelt findet sich $\partial^{2} \rho$ in $d \partial^{2} s x(t a c)$, vgl. $\$ 30,1$, aber adverbiell fyurdu'ax (vor tag), ferner in $m \partial^{2} o n$ (man), vgl. $§ 31,4, g r \partial^{2} J m$

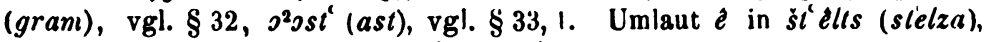
aber consequent $a^{2} a$ in $b \rho^{2} s x$-št $a^{2} a l t s k^{2} n$ (bachstelze, wazzarstelza).

2. Vor $m+\operatorname{con} 8 ., \quad m m$ : ḱ $0^{2} \mathrm{Jm}$ (chamb), lo2 Jm (lamb),

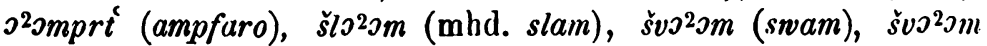
(prät. von swimman), ḱ ${ }^{2} 3 n ı p / r$ (mbd. kampfer); u mlaut: k' $e^{2} m a n$ (chemben), le ${ }^{2} m r n$ (lämmern), he ${ }^{2}$ man (*hemmen).

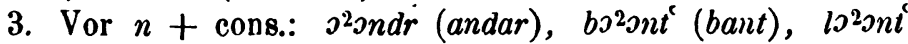

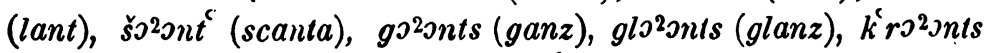

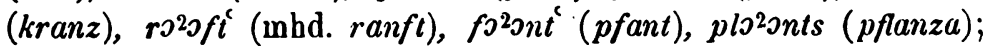
umlaut: a) vor $n p, n d$ : frońdrn (verändern = verheiraten), bəhońt (bihenti), ońt (enti), lońdn (pl. v. lenti), šońdn (scenten); b) vor $n t$ : bak'ri²ntsn (bekränzen), pli²ntsk'n (pflïnzlein). 


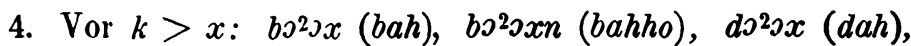
‘' ${ }^{2}{ }^{2} x l$ (chahhala). Umlaut: hêxl (mhd. hechel), rêxnon (rehharôn).

§ 30. Häufig erscheint aites $\breve{a}$ als $u^{2} \partial$; und zwar ist dies die regel vor einfachem stimmhaftem dauerlaut, ausgenommen die nasalen. - Als stimmbafte dauerlaute kommen in der mundart binzu $z, v, z$ aus altem $s, b, g$. Das $u^{2 a}$ findet sich fernerhin vor altem $r m, r n, r d, r s$, vor $h s$ und $h t$. Der umla ut von diesem $u^{2} \partial$ ist $\hat{a}$, nur vor rm $a^{2}$. - Beispiele:

1. Vor $l, r, s, b, g: t s u^{2} a l$ (zala), šu al (scala), zu'alvet't (salaha, weide), mu²aln (malan); špuªrn (sparôn), fü2arn (faran),

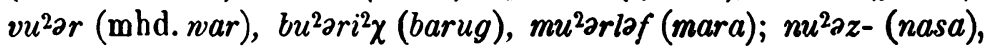
glu2az-(glas), hu2az-(haso), füa-zln (fasôn); nuªvl (nabulo), šuªvn (scaban), gru'2avn (graban), gru²avn (grabo); nu'2azl (nagal), tsuazl (zagal), vuªzn (wagan), $h u^{2} \partial \bar{z}^{2}$ (hag). - Umlaut: šêelí $\chi^{n}$ (schälchen), špérzm (sparsam); grâez- (gras), glâez(trinkglas; vgl. oben glu²az-glas im allgem.); grôev-(grab); nốjln (nageln).

2. Vor rm: vuiarm (warm), u2arm (arm adj.); umlaut: vae $r m a n$ (warmen), a $e^{2} r m r$ (comp. v. aram).

3. Vor $r n, r d, r s ; h s, h t$ : gu²arn (garn); šene $2 a r t t^{c}$ (*swarta),

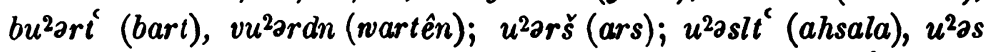

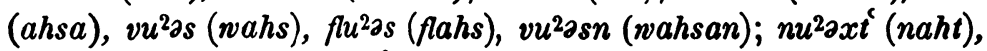
vu2axtl (wahtala), $u^{2} \partial x t^{t}$ (ahta). - Umlaut: êrnon (arnôn);

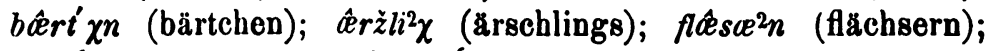

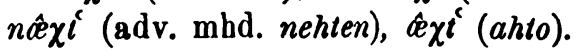

A $\mathrm{nm}$. Sonst findet sich $u^{2} \partial$ noch in $b u^{2} a t^{t}(b a t), s^{2} u^{2} \partial t^{t}$ (adverbiell, subst. šadn, scado), fü2tr (fater - dagegen gofadr = gifatero)

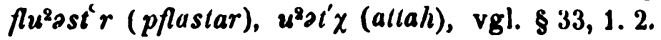

$\S 31$. Westgerm. a wird zu $0^{2}$ vor altem $n g>n$, resp. $x k$, vgl. $\S 106$, und vor altem $n k>v k ; o^{2}$ findet sich ferner vor $n n$. Umlaut: vor altem $n g: o(n)$, vor $n k: i^{2}(n k)$, vor $n n: e^{2}$. Beispiele:

1. Vor $n g>n: o^{2}$ bl (augel), $o^{2}$ rast (angust), $b 0^{2} x$ (*bango), ślo ${ }^{2}$ s (slango), lo²rón (langôn). Umlaut: drońan (drengen), oń (engi), moñan (mengen), zoñan (mhd. sengen), šlońln (schlängeln).

2. Vor $n g>n k^{k}: l 0^{2} n k^{l}$ (lang), štro ${ }^{2} n k^{k}$ (strang). Umlaut: štroń (pl. von strang).

Anm. Eigentlich sollten, wie im pl. štroń, alle $n g$ welche nach 
$\S 106 \mathrm{zu} \otimes k^{k}$ werden, mouilliert erscheinen, da wenn umlautung erfolgt, ng ursprïnglich geschiit $\%$ sein musste; doch lautet der comparativ von $l 0^{2} n k$ (lang) lez $e^{2} k^{2} r$.

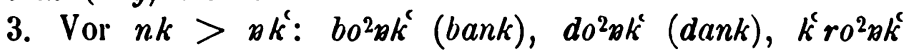
(krank). Umlaut: di ${ }^{2} n k n$ (denchen), li ${ }^{2} n k^{k} n$ (mbd. lenken),

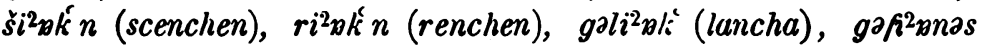
(mhd. gefencnisse).

4. Vor $n n>n$ : k $o^{2} n$ (channa), špo ${ }^{2} n$ (spanna), fo ${ }^{2} n$ (pfanna). Umlaut: k $e^{2} n a n$ (chennen), re ${ }^{2} n a n$ (rennen), fe $e^{2} n a x k$ (pferining).

$\mathrm{Anm}$. $o^{2}$ findet sich auch in $k^{k} l o^{2} m r\left({ }^{*}\right.$ klamara), und šro ${ }^{2} m$ (mhd. sram, -mml-). Umlaut $e^{2}$ in he $e^{2} m \imath^{c}$ (hemidi).

$\S 32$. Altes $\bar{a}$ wird zu $u^{2}$ vor einfachem altem $m$ und $n$. Umlaut von diesem $u^{2}$ ist $i^{2}$. - Beispiele: $h u^{2} m r$ (hamar), hu²ml (hamal), zu'mln (samanôn), šu ${ }^{2} m \partial n$ (scamên), nu²man (namo),

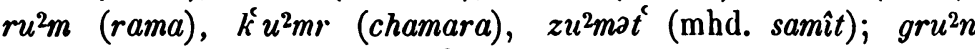

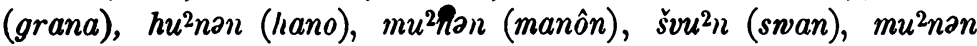

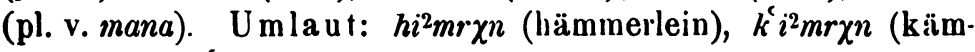
merlein); i2nt (anut, enit).

$\S 33$. Altes $\breve{a}$ erscheint als $a$ regelmässig vor altem $t, p$; $t t, p p, k k ; s p, s t, s k, f t$ - also vor stimmlosen lauten und lautgruppeu; ferner vor altem $b, d ; r t, r p, r k-r b, r g, r w-$ $r r$, ll. - Beispiele:

1. Vor $t, p ; t t, p p, k k ; s p, s t, s k, f t: v a s r$ (wazzar), nas (naz), gas (gazza); zaft (saf); ḱats (chazza), ḱratsn (chrazzôn), rats (rato), glats (mhd. glatz); tsapn (zapfo), apl (apful), ḱap (chappa); šmaḱn (smucchên), hak'n (*hacchôn), ak'rn (zu ucchar), bakin (bacchan), fakl l (facchala); gasi (gast), fastn (fastên), haspl (haspil), flaš (flasca), našn (nascôn), k'raft (chraft).

2. Vor $b, d$ : lat (*lada), ladn (hladan und ladôn), mat (mado), šadn (scado), fadm (fudum), fladn (flado); gafadr (gifatero), zat (sat), špadl (*spato), ḱadr (chataro).

3. Vor $r t, r p$, rk: švarts (swarz), varts (warza), šarf (scarf), št ark' (stark).

4. Vor $r b, r g, r w$ : garf (garba), arג (arg), farf (farava).

5. Vor rr, ll: dar (darra), far (pfarrâri), ḱar (charra); al- (al), faln (fallan), ši al (stal), gal (galla).

Anm. Den bisher gefundenen regeln widersprechend findet sich $a$ in folgenden fällen: gafl (gabala), hasl (hasala), vgl. §30, 1; avr (abur), arm (arm, subst.), hart (herti), k'aro-fret'tu'x (chara-freitag), vgl. 
§30, 1. 2. 3; gald'jn (galgo), half (halb), halls (hals), zalf (salba), švalf swalawa); sitamipn (stampfön), síam (stam), švax (swach); laxn (lahhen), zax (saliha), vgl. §29, 1.2. 4.

§ 34. Entsprechend der mannigfaltigkeit der laute, vor denen altes $\breve{a}$ sich als $a$ erhalten hat, ist auch die menge von hierhergebörigen umlauten.

1. $\hat{e}$ erscheint consequent vor ursprunglich doppeltem verschlusslaut: $\hat{a} t s n$ (ezzen), lâtsn (lezzen), zêtsn (sezzen), vôetsn (vezzen), gazcêts (gisezzida), ḱvêttšn (mbd. quelschen), ḱk âtsḱkn (dem. von chazza); šnôp (snepfo), šếpn (scepfen), trếp (mhd.

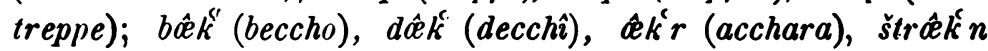

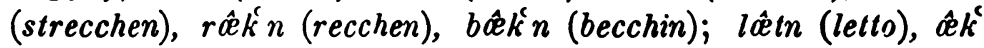
(ekka), šnôkk (snekko).

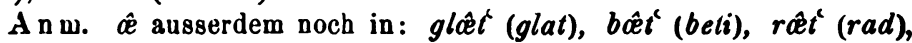
šcêdu (scato), fột $t^{t}$ (pl. von $f u^{2} \partial t^{i} r=$ fater).

2. $a^{2}$ findet sich vor $p, d,++$ cons., $r r: f a^{2} r t^{2} i^{2} \chi$ (fartíg),

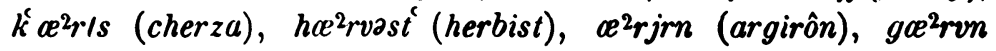
(gariven), $e^{2} r b a s$ (araweiz), pac ${ }^{2} \gamma \chi$ (pferrich); $a^{2} d l$ (edili), fla $a^{2} d n$ (pl. von flado), fatir (fetiro).

A nm. $a^{2}$ ferner in $e^{2} r n$ (ero), dce $c^{2} n$ (tenni); hierher gehört vielleicht auch maentiš (mennisco), mit vor $\ddot{n}$ geschlossenem $\alpha$.

3. $a^{2} a$ ist der umlaut des $a$ vor $l l: g a-z a e^{2} a l$ (gisello), $h a^{2} a l$ (hella), ši $a^{2} a l$ (stella).

4. $i^{2} \partial$ ist der umlaut der ubrigen $a$. $-i^{2 \partial}$ scheint der ursprunglichste umlaut von altem $\bar{a}$ zu sein, denn es findet sich in wörtern, welche nur umgelautete formen aufweisen auch vor solchen lauten, welche sonst eine der bereits behandelten palatalisierungen fordern; z. b. hi2alt (halda), $i^{2} a l i r$ (alturi), vgl. \$ 29, 1, iłazl (esil), i2al (elina), liªjn (legen), $z i^{2} a j$ - (sega), miªr (meri), k'i2arn (cherian), fli ${ }^{2} e j l$ (flegil), vgl.

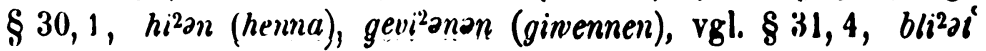
(blat), aber pl. blac ${ }^{2} d r, \mathrm{vgl}$. $\S 34,2$.

Regelmässig steht $i^{2} \partial$ vor $p, t, s t, s k, f t: l i^{2} \partial f l(l e f / i l), \hbar^{2} a s l$ (fezzil), biªsr (bezziro), mi2asr (mezziras), k' ${ }^{2} 2 s l$ (chezzil), niªsl (nezzil), li²atst (lezzist); fiªst (festi), riªstn (resten), gizasi (pl. von gast), vi2ašn (wascan), fliªšk n (fläschchen); gaši ${ }^{2} \partial f t$ (geschefte mhd.), k'ri ${ }^{2} \partial f t i^{2} \chi$ (whd. kreftec).

$\S 35$. Zu betrachten sind noch die fälle, wo durch ausfall eines auf altes $\breve{a}$ folgenden lautes contractionslänge entstanden ist. 
1. $\hat{o}$ findet sich, wenn altes $h$ oder $g$ nach $\not{a}$ ausgefallen ist und kein umlauterzeugender vocal nachfolgte; war letzteres der fall, erscheint $\hat{e}$. Beispiele: šlôn (slahan), šlôl (stahal); hôl (hagal), k'lôn (chlagôn), zôn (sagên), ônan (pl. v. agana). Umlaut: êr (ehir), tsêr (zahar), gavênan (yiwahinen); ế (egida), êdos (egidehsa), mêt (magad, pl. megidi), êltstr (agalstra), gênat (mhd. gegenôte).

2. Nach abfall von $w$ findet sich $y u$; der umlaut dieses $y u$ ist $\hat{\imath}$; der umlaut $o^{2} \jmath^{2}$ hat sich in wörteru entwickelt, welche nur umgelautete formen haben. - Beispiele: fryu (frô), umla ut: a) frîli ${ }^{2} \chi$ (fröblich), štri (strô), b) štro $0^{2} \jmath^{2}$ (strewen), fro $0^{2} \jmath^{2} \hat{t}$ (frevida), fardo $0^{2} J^{2}$ (-dewen), ho $o^{2}$ (hewi).

Anm.1. -aw- ist wahrscheinlich mit altem au zusammengegangen, vgl. $\$ 77,2$; -ew- wahrscheinlich mil altem -iun-, vgl. $\S 83$.

A nm. 2. Das $o^{2} \rho^{2}$ in $g o^{2} \rho^{2} s$ (gans) und $h o^{2} \rho^{2} s l^{\prime}$ (hengisl) ist sicher - im gegensatz zum $\partial^{2} \mathrm{o}$ in ro ${ }^{2}$ oft (mhd. ranft), vgl. $\$ 29,3$, - umlautsvocal.

2. Westgerm. $\check{e}$.

Am weitaus häufigsten entspricht altem $\check{e}$ im dialekt $i^{2} \partial$; es verhält sich mit diesem $i^{2} \partial$ ähnlich, wie mit dem gleichlautenden umlaut von $\breve{a}$ (vgl. $\S 34,4)$; der ubersichtlichkeit wegen sind deshalb die consequenteren entsprechungen von westgerm. $\check{e}$ vorangestellt.

§ 36. Altes $\check{e}$ wird zu $c^{2} a$ vor altem $l+$ cons. und $l l$, (vgl. $§ 29,1 . \S 34,3$ ). Beispiele: far $^{2} a l t^{\prime} \chi$ (fëlga), foe ${ }^{2} a l l s$ (fë-

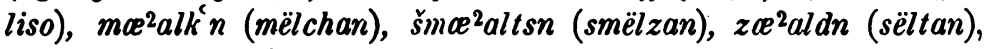

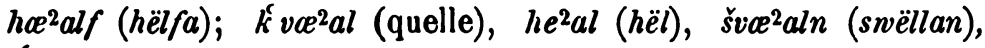
$k^{r} a^{2} a l r$ (chëllari).

$\S 37$. Altes $\check{e}$ wird zu $a^{2}$ vor altem $b, d, r+$ cons. (ausser $r s$ ), $r r$ (vgl. § 34, 2), ferner vor $p$ und st. Beispiele:

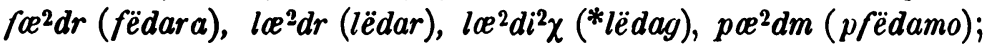

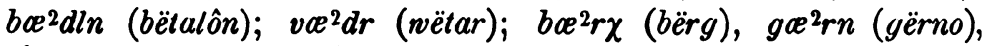
$k^{\prime} a^{2} r n$ (kërno), vae $r k$ (wërah), k' $a^{2} r v n$ (mhd. kërben), ši $a^{2} r v n$

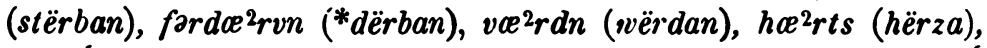

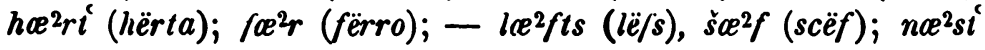
(nëst).

$\S 38$. $\check{e}$ erscheint als $\hat{e}$ vor altem $k>$ (in der mundart

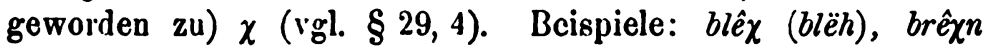




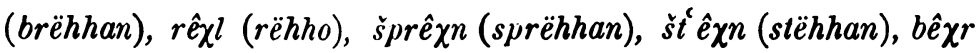

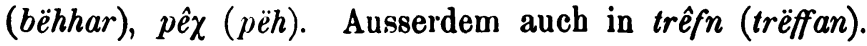

$\S 39$. $\breve{e}$ wird $z u \hat{e}$ vor altem doppeltem verschlusslaut (vgl. § 34,1). Beispiele: $\hat{x} t s l i^{2} \chi$ (ëtteslîn), ḱk lát (chlëtta), flâk̂́n (flëccho), lộḱn (lëcchôn).

$\S 40$. In allen ubrigen fällen ist altes $\check{e}$ zu $i^{2} e$ geworden. Beispiele: giªl (gëlo), mi2al (mëlo); hi²ar (hëra), šviªrn (swëro), fiªrš (fërsana), giªršt (gërsta), piªrš (mbd. pfërsih); i2avn

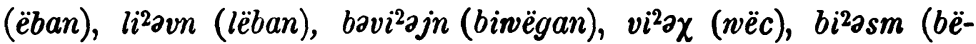
samo), li2azn (lësan); i²asn (ëzzan), mi²asn (mëzzan), k'i2avr (chëvar), šli²a $t^{\dagger}$ (slëht), zi²as (sëhs), driªšn (drëskan).

A n m. 1. iz steht ausserdem noch in folgenden fällen, welche mit dem in $\$ 36.37$ bemerkten in widerspruch stehen: giºlt $(g e l t), f^{2} a l t^{6}$

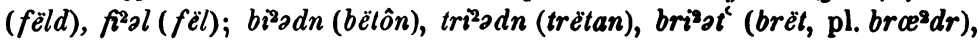
$i^{2} a r t^{\prime}$ (ërda), švizart' (swërl), hìart' (hërd).

A nm. 2. Als $i^{2}$ erscheint altes $C$ ausnshmsweise in folgenden

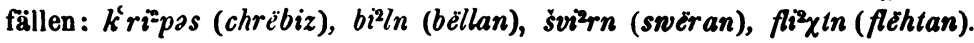

§ 41. Als contractionslängen erscheinen:

1. $\hat{e}$ nach ausfall von altem $g$. Beispiele: rên (rëgan), zênts (sëgansa).

2. Âe nach ausfall von altem $h$. Beispiele: gašên (giscëhan), zồn (sëhan), tsôen (zëhan), fồ (fëhu).

3. Westgerm. $\check{\imath}$.

$\S$ 42. In den meisten fällen erscheint altes $\breve{\imath}$ als $a^{2}$, nämlich vor allen westgerm. verschlusslauten, welche im dialekt stimmlos sind (ausgenommen also vor den einfachen alten $b, d, g$ ), ferner vor $s t, s c, s s, f t$, also wider vor stimmlosen lauten. Endlich steht $a^{2}$ vor $m+$ cons, $m m, n n$ und $n k>n k$. Beispiele:

1. Vor stimmlosen lauten: varsn (nizzan), st $a^{2} x$

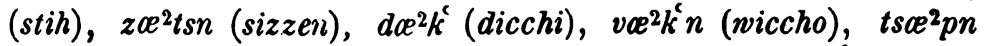

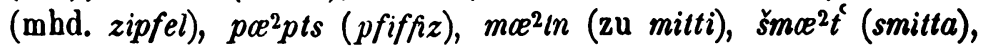

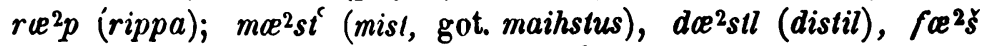

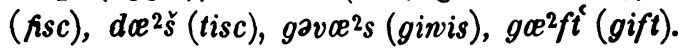

2. Vor $m+$ cons., $m m, n n, n k>n k^{c}: \check{s}^{2} m l$ (*scimbal),

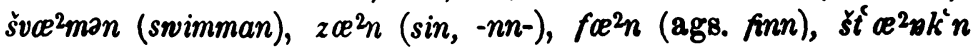

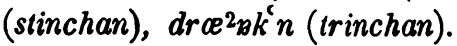

Anm. $a^{2}$ findet sich auch in $z e^{2} f(s i b)$, wahrscheinlich wegen steter stimmlosigkeit des $f$ in diesem worte. 
$\S 43$. Vor $l+$ cons. und $l l$ ist altes $\breve{\imath}$ in $a^{2} a$ ubergegangen (vgl. § 36). Beispiele: vae alt (wildi), vce aln (willo), št $\mathfrak{c}^{2} a l$ (stilli), dae al (tilli).

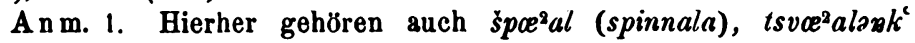

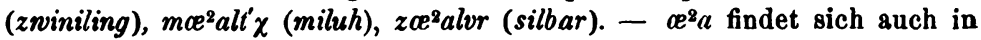
spocal (spil) und $\grave{s}^{2} \mathfrak{c}^{2} a l\left(s t i{ }^{2}\right)$ in der bedeutung 'stiel an axt, hacke'; in allgemeiner bedeutung 'stiel' aber 'šti l, welches wort mit šl' $a e^{2} a l$ nicht in zusammenhang gebracht wird.

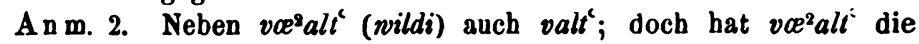
speciellere bedeutung wild, wildbret.

$\S 44 . i^{2}$ und $e^{2}$ als entsprechungen von altem $\breve{\imath}$ greifen vielfach in einander uber, doch erscheint $e^{2}$ meist vor $r+$ cons., $r r$ und $t$-lauten, $i^{2}$ weitaus am häufigsten vor stimmhaften spiranten, also vor altem $s, b, g$, ferner vor einfachem l, r. Beispiele:

1. $\check{\imath}>e^{2}$ vor $r+$ cons., $r r, t$-lauten: gabe ${ }^{2} r \chi$ (gibirgi), he ${ }^{2} r n$ (hirni), št é $e^{2} r n$ (stirni), ve $e^{2} r v l$ (wirbil), gaš $e^{2} r$ (giscirri), $e^{2} r$

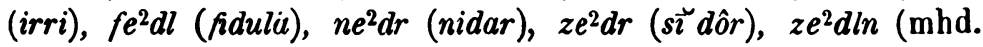
sidelen), tse ${ }^{2} d r n$ (zittarôn).

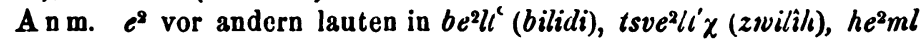
(himil), le $e^{2} l^{\prime} \chi$ (lilja).

2. $\check{\imath}>i^{2}$ vor $s, b, g, l, r: v i^{2} z$-(wisa), vi2zl (wisala), gi2vl (gibil), zi $i^{2} v n$ (sibun), ri ${ }^{2} j l$ (rigil), švi ${ }^{2} j r$ (swigar), bi ${ }^{2} r$ (bira), $k^{2} i^{2} r \chi$ (chirihha), $\AA^{2} l$ (filu), špi2ln (spilôn).

A n m. vor anderen lauten: $b i^{2} d n$ (bitten), šliz $d n$ (slito), fri $i^{2} d n$ (fridu), dialn (dillo).

§ 45. Eine besondere behandlung erfährt altes $\breve{z}$ in den gruppen -ing- und -inp-, -ind-. In der gruppe -ing-geht es in $-a(\dot{n})$ - uber, in den beiden andern in $-a(\dot{n} d)$ - oder -ce(nd)-, und zwar in $-a(\dot{n} d)$ - dann, wenn die gruppen durch folgenden rocal geschutzt waren, in - $\propto(\dot{n} d)$-, wenn sie auslauteten. Ausgleichung hat zumeist in der art stattgefunden, dass bei nominibus der nominativ, bei verbis der infinitiv den ausschlag gegeben hat. Beispiele:

1. -ing-: drañan (dringan), fańr (fingar), galañan (gilingan), zañan (singan).

2. -inp-, -ind-: a) bańdn (bintan), fańdn (findan), gəšvańt (*swindo), gazańt (gisindi), lańt (linta), rańt (rinta); b) ḱceńt (chind), grańt (grint), vañi (wint), zañt (mbd. sint, sit $)$. 
A nm. 1. dón (ding) neben dañon (mhd. dingen) deutet auf eine zu -ańd-:- $e^{2} r i d$ - parallele entwicklung der gruppe -ing- hin.

A n m. 2. varńt $r$ (vintar) scheint sich an voent (wint) angeschlossen zu haben. In blant' (blint) und rañ' (junges rind) ist von den obliquen casus aus ausgeglichen worden; neben rañt ateht aber das compositum roeñtsflišs (rindfleisch).

$\S 46$. Als $\hat{\imath}$ erscheint altes $\breve{\imath}$ wenigemal, in hîrs (hirsi), tsvîrn (mhd. zwiren), hît (hirti) neben hỉrt.

$\S 47$. Altes $\breve{\imath}$ erscheint als $o^{2} \jmath^{2}$, wenn ein darauf folgender nasal vor $s, f$ ausgefallen ist. Beispiele: $d 0^{2} v^{2} z n$ (dinsan), $l 0^{2} \partial^{2} z n$ (pl. v. linsi), $0^{2} \partial^{2} s l t$ (unslit, mhd. inslit), tso $0^{2} \partial^{2}$ (zins), $f 0^{2} \partial^{2} \operatorname{stn}\left({ }^{*}\right.$ pfingustin), $f 0^{2} \partial^{2} f$ (finf). Ebenso nach ausfall von altem $g(g g)$ in $l 0^{2} 0^{2} n$ (ligen).

4. Westgerm. $\check{~}$.

$\S 48$. Altes $\breve{o}$ erscheint in den meisten fällen als $\hat{o}$, ganz regelmässig nämlich vor altem $p, k ; t t, p p, k k ; p b[d d, b b], g g$, also vor consonanten, die jetzt im dialekt alle stimmlos sind: $f, x ; t, p, k ; t[t, p] k$. Der umlaut dieses $\hat{o}$ ist $\hat{e}$. - Beispiele: ôfn (offan), lôx (loh), ḱlôts (mhd. kloz), hôp (hopfo), lôk'n (locchôn), môt (mhd. molte), rôk'n (rokko). Umlaut: št êk'n (die reben mit 'stöcken' verseben), špêtln (spötteln).

$\S 49$. Altes ó wird zu $u^{2} \partial$ vor $t$, st $[s p]$, sk, ss. Der umlaut hiervon ist $i^{2} \partial$. Beispiele: šlüas (sloz), gašù asn (scozzan),

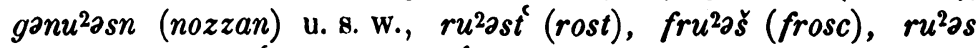
(ros, -ss-), mu2asi (most), fü2ast (pfosto). - Umlaut: fri2aš (pl. v. frosc), rǐ̌sk' $n$ (rösslein).

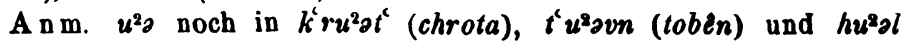

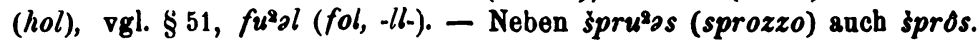

§ 50. Vor $l+$ cons. ist westgerm. $\check{o}$ in $\hat{u}$ ubergegangen. Der umlaut hierzu ist $\hat{\imath}$. Beispiele: št alls (stolz), falk (folk), valkin (pl. v. wolcha), vâlof (wolf). Umlaut: v̂ləof (pl. von wolf), fîlk r (pl. von folk).

A $\mathrm{n} m$. $\hat{a}$ findet sich auch in dîxt $r$ (tohter).

$\S 51$. $\mathrm{Zu}$ yu wird altes $\check{o}$ vor einfachem stimmbaftem dauerlaut: $l, r ; b, g, s, f>v, z, z, v$; ferner vor altem $r n$ und $r d$. Der umlaut $\hat{\imath}$ ist einigemal zu $e^{2}$ und $i^{2}$ verkurzt. Beispiele: ḱyul (cholo), zyul (sola), byurn (borôn), k'yurn (chorôn), dyurn (dorn), hyurn (horn), byurtn (borto), vyurt (wort); lyuf (lob), byuzn (bogo), hyus (hosa), yuvn (ovan). Umlaut: 
a) $u^{2} n$-hîzln (hosen anlegen), îvn (pl. von ovan) etc. etc., mîrí rt (mhd. mortel, mortarium), ebenso mîržl (morsâri, mortarium), hîš (mhd. hövesch); - b) de 2 rnar (pl. von dorn), ze 2 ln (solan); c) $f^{2} z l$ (pl. von fyuzl = fogal).

An m. Altes $\partial$ ebenfalls zu yu geworden in byuršt (burst) und yus (ohso).

$\S 52$. Altes $\check{o}$ findet sich als $o^{2}$ vor $b, d, r+$ cons. ausser $r n$ und $r d$ (vgl. §51). Der entsprechende umlaut ist $e^{2}$. Beispiele: $b o^{2} d m$ (bodam), k'no $d n$ (chnodo), do ${ }^{2} d r$ (totoro), go $0^{2} t^{6}$ (got), do ${ }^{2} r f(d o r f), f o^{2} r \chi t$ (forhla), mo²rjn (morgan), $k^{2} o^{2} r f$ (chorp). Umlaut: af-kne $n e^{2} d r n$ (knoten lösen), tsaa-be $e^{2} d a m a n$ (ein gefäss mit einem zweiten boden schliessen), de $2 r f r$ (pl. von dorf), fer $e^{2}$ tn (furihten).

A n m. 1. $o^{2}$ erscheint auch in $k^{k} n o^{2} b l a n k$ (chlobolouh), hof $n$ (mhd. hoffen), $o^{2}$ pas (obaz), ho²naxk, ältere (?) nebenform von hont' $\chi$ (honag), fold'jn (folgên).

Anm. 2. An andern vereinzelten entsprechungen von altem $ठ$

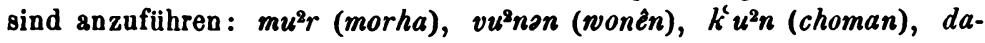
nar (donar).

Westgerm. $\breve{u}$.

§ 53. Altem $\breve{u}$ entspricht $a$ vor den stimmlosen $t, p, s t$, $s k, f t>s, f, s t, s, f t$; ferner vor altem $n n, m m$, und $m+$ cons. Der entsprechende umlaut ist $a^{2}$. - Beispiele:

1. nas (nuz), flas (fluz), bat (butina), bat'r (butera), haf (huf), k'afr (chupfar), last' (lust), brast (brust), laft (luft); umlaut: šloe $2 s l$ (sluzzil), ša ${ }^{2} s l$ (scuzzila), bae fl (mhd. büffel),

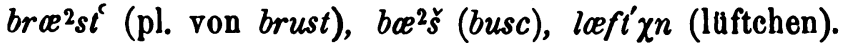

2. zan (sunna), branon (brunno), št am (stum, -mm-), zamp (sumpf), rampln (mhd. rumpeln), ḱ lampn (ndd. klump); umla ut:

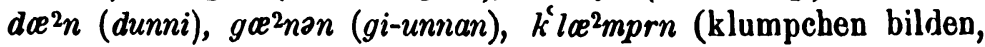
intransitiv).

$\S 54$. Altes $\breve{u}$ ersoheint als $u^{2}$ regelmässig vor $r+$ cons., einigemal auch vor $m+$ cons. (vgl. $\S 53$ ). Beispiele: 1. $f u^{2} r$

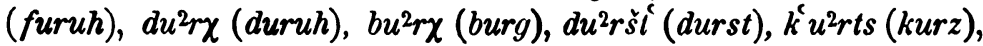

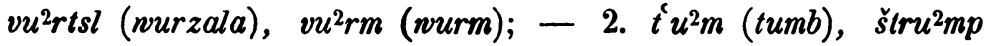
(mbd. strumpf).

Der umlaut dieses $u^{2}$ ist teils $e^{2}$, teils $i^{2}$; doch scheint $e^{2}$ der ursprunglichere umlaut zu sein, da es sich in wörtern findet, welche durch alle formen umlaut haben mussten. Bei- 
spiele: a) be ${ }^{2} r t$ (burdi), fe ${ }^{2} r d r$ (furdir), i $e^{2} r f n$ (durfan), ve ${ }^{2} r j n$ (nurgen); - but $r \chi$, be $e^{2} r j r$ (buirger), kurts, comp. k $e^{2} r t s r$. -

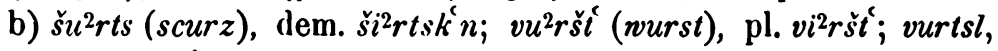
dem. vi²tsli $\chi n$.

A n n. Fernerhin findet sich $u^{2}$ in $f u^{2} s$ (fuhs), $t r u^{2} t$ (mhd. trute), frute $t^{2}$ (fructus).

$\S 55$. Als $o^{2}$ erscheint altes $\breve{u}$ vor $m, n$. Der entsprechende umlaut ist $e^{2}$. - Beispiele: $f 0^{2} m$ (mhd. vrum), zo ${ }^{2} m r$ (sumar), $1 o^{2} n$ (luna, aclisennagel); umlaut: fre ${ }^{2} m r$ (comp. zu fro ${ }^{2} m$ ),

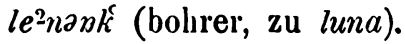

$\S 56$. Altes $\breve{u}$ wird zu $\hat{u}$ vor $l+$ cons. (vgl. § 50). Der umlaut ist $\epsilon^{2} a$. Beispiele: dûldn (dulten), gadolt (gedult), šult (sculda), šâldr (scultara), gâlí (golt); umlaut: gadoe ${ }^{2} a l d i^{2} \chi$ (mhd. gedullec), ša $e^{2} a l d i^{2} \chi$ (mhd. schuldec), gre ${ }^{2} a l d e^{2} n$ (guldîn), foc aln (fullen).

$\S 57 . a^{2} a$ aus altem $\breve{u}$ findet sich consequent vor altem $n g, n k, n p, n d, k>x$. Diesem $a^{2} a$ entspricht verschiedener umlaut: vor altem $n g>n: a(\dot{n})$, vor $n p$, $n d>n d: a(\dot{n} d)$; in den übrigen fällen lautet er $a^{2}$. Beispiele:

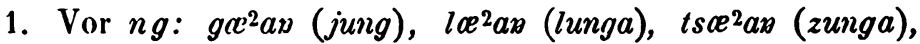

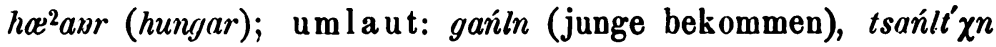
zitnglein.

2. Vor $n f, n d: f e^{2} a x d n$ (part. von findan), grae axt

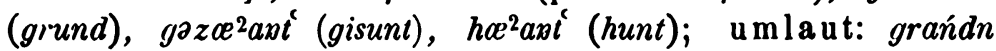
(gründen), zañt (sunta), hańt $\chi^{n}$ (hündlein).

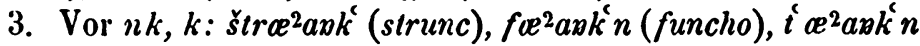
(tunchôu); bra ${ }^{2} a x$ (bruh), špracax (mbd. spruch); umlaut: $f a^{2} x k^{k}$ ln (funkeln), štr $a^{2} n k^{k}$ (pl. zu strunc), br $\alpha^{2} \chi, \check{s} p r a^{2} \chi$ (pl. zu bruh, spruch).

§58. Da altes $\breve{u}$ häufig unter dem schutz eines $i(j)$ in folgender silbe stand, so sind die umgelauteten entsprechungen desselben am zablreichsten.

1. Altes $\breve{u}$ erscheint als $a^{2}$ vor altem unter einfluss von folgendem $j$ verdoppeltem $t t, p p, k k ;[b b, d d, b b], g g$. Beispiele: îl $c^{2} t s i^{2} \chi$ (einluzzi), št $a^{2} t s n$ (stuzzen), ḱn noe ${ }^{2} p n$ (knupfen),

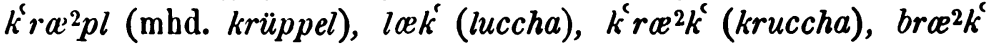
(brucca), maek (mucca).

2. Als $i^{2}$ erscheint altes $\breve{u}$ vor $l, r, b, g$ mit folgendem $i$. Beispiele: $m i^{2} l$ (muli), di ${ }^{2} r$ (turi), šni ${ }^{2} r \chi\left({ }^{*}\right.$ snurihha), $i^{2} v l$ (ubil), 
$i^{2} v i^{2} \chi$ (mhd. überic), li ${ }^{2} j n$ (lugina), fli2jl (mhd. vlügel), $p i^{2} l$ (pfuliwi).

A n m. $e^{2}$ findet sich in $f e^{2} \ln$ (fulîn), $m e^{2} \ln r$ (mulinâri). - Das $i^{8}$ in $d i^{2} x k^{2} n$ (dunchan) ohne zweifel folge von anlehnung an $d i^{2} x k i n-$ denchen § 31,3 . - Sonst steht $i^{2}$ noch in bia (buhsa), $k^{2} i^{2} m$ (chumil).

§59. Altes $\breve{u}$ wird zu $c a$, wenn darauf folgendes $n$ vor $s, f$ geschwunden ist: acas (uns), farnoaft (firnunft). Ausserdem steht ea auch in $a a(n)-, u n-$, z. b. $a a-n \hat{\imath} d i^{2} \chi$ (unnötig).

\section{b) Lange rocale.}

1. Westgerm. $\boldsymbol{a}$.

$\S 60$. Westgermanisch $a$ erscheint in der mundart regelmässig als $\hat{o}$, nur vor $h t$ als $u^{2} \partial$. Der umlaut ist im allgemeinen $\hat{e}$ (vgl. § 48), aber $\hat{a}$ vor ht (vgl. § 30,3). Beispiele:

1. ôl (ala), hôr (hâr), zôm (sâmo), môn (mâno), ôvnt (aband), ôdr (adara), frôzn (frâgên), môs (mâza), brôx (brâhhha), hôk'n (hâko), blôs (blâsa); umlaut: mêr (mârî), mênat (mânôl), grêf (grâvo), gafês (gifazzi).

2. $u^{2}$ nduaxt (anadâhl), duəxt (tâht); umlaut: $u^{2} n d \hat{e} \chi t^{t} i^{2} \chi$ (andäcbtig), dếxt (pl. von tâht).

A n m. $u^{2} \partial$ findet sich vereinzelt in $m u^{2} \partial x t^{t}(m a g o), m u^{2} \partial t^{\imath}(m a d)$, rutest (mhd. raze).

$\S 61$. Die lautgruppe $-\hat{a} w-[-\hat{a} h-]$ zeigt sich als $\hat{o}$; umlaut $\hat{e} ;-\hat{j} j$-erscheint als $\hat{e}$. Beispiele: blô (blâo), ḱk̂ (chlawa), fèn (fâhan)', hên (hâhan), tsè (zâhi), ‘jề (gâhi), bên (bâjan), zên (sajjan).

2. . Westgerm. $\hat{e}$.

§ 62. Altes $\hat{e}$ erscheint teils als $\hat{e}$, teils als $\varkappa^{2}$; und zwar letzteres vor altem $g, k$. Beispiele: 1. hé (hiar), šâr (sciaro),

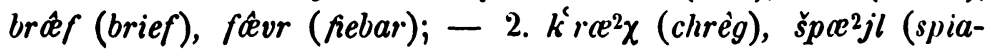
gal), $t s a^{2} j l$ (ziagal), tsae ${ }^{2} \chi(z i a h h a)$.

3. Westgerm. $\hat{\imath}$.

$\S 63$. Westgerm. $\hat{\imath}$ erscheint vor alten $p, d, n$ (welche mouilliert werden, vgl. \$§ 90. 96. 103) als $e$ : šned dn (snîdan), net'i (nî̀d), šred dn (scrîtan), šet í (scit), k'ret't (krîda); šerian (scînan), šveń (svîn), feñ ( fîn).

Anm. Das $i^{2}$ in ant-k $k^{2} i^{2}$ an (kînan) ist ohne zweifel aus dem participium (gešiznan - ahd. scinan) herübergenommen.

$\S 64$. Als $e^{2}$ tritt altes $\hat{\imath}$ auf vor altem $l, m, k, g: k^{\imath} e^{2} l$ 


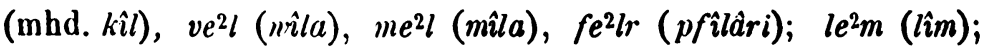
šle ${ }^{2} \chi n$ (slihhan), gle ${ }^{2} \gamma$. (gilîh), ve ${ }^{2} \chi n$ (wîhhan), $r e^{2} \chi$ (rîhhi); šve $e^{2} j n$ (swîgên), ši $e^{2} j n$ (stîgan), ge $e^{2} \chi$ (gîge mhd.).

A $n$ m. Vereinzelt findet sich $e^{2}$ in $g e^{2}\left(s i^{2} \chi\right.$ (gîlag); ferner in šle ${ }^{2} s n$ (slîzzan), wahrscheinlich in folge von anlehnung an šle $e^{2} s n=$ ahd. sliozzan.

$\S 65$. In allen ubbrigen fällen ist altes $\hat{\imath} \mathrm{zu} e^{2} e$ geworden: $v e^{2} e f$ (wîp), $r e^{2} e f(r \hat{\imath} / \hat{i}), b e^{2} e s n$ (bîzzan), $e^{2} e z n$ (îsan), ki le $e^{2} e s i \hat{r}$ (*chlistar), kire ${ }^{2} e \grave{n} n$ (*chrîskan), t' $e^{2} e s l t$ (dîhsala), fe ${ }^{2} e f$ ( $\left.p f \hat{\imath} f a\right)$, $f e^{2} e r$ ( fîra).

$\S$ 66. Die verbindungen -îj-, $-\hat{\imath} w-$, $-\hat{\imath} h-$ auslautend und mit folgendem vocal werden $\mathrm{zu} o^{2} \jmath^{2}: b o^{2} \jmath^{2}(b \hat{\imath} a), d r o^{2} \jmath^{2}(d r \hat{\imath})$,

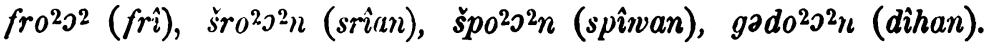

4. Westgerm. $\hat{o}$.

$\S 67$. Westgerm. $\hat{o}$ erscheint vor altem $k, g>x, z$ als $a^{2} a$. Der entsprechende umlaut ist $a^{2}$. Beispiele: $b a^{2} a x$ (buoh), š $\alpha^{2} a x$ (scuoh), kil $l a^{2} a x$ (kluog), k'ra $a^{2} a x$ (kruog). Umlau t: $d a^{2} \chi r$ (pl. von tuoh), foe ${ }^{2} j n$ (fuogen); $z a^{2} k^{k} n$ (suohhen).

§ 68. Als $o^{2}$ erscheint aites $\hat{o}$ vor $t, p ;[d d], b b ; s t>s, f ;$ $[t], p$; st. Der unlaut ist $e^{2}$. Beispiele: $b^{2} s$ (buozza), fo ${ }^{2} s$ (fuoz), ro ${ }^{2}$ fn (ruofen), ho2stn (huosto). Umlaut: ze's. (suozzi), $m e^{2} s n$ (muozzan); re $e^{2} p$ (ruoba), he $e^{2} s t$ ln (husteln).

$\mathrm{An} \mathrm{m} . o^{2}$ findet sich auch in $m o^{2} t^{t} r$ (muoter) und $h o^{2} t^{\prime}$ (huot), wol darum, weil altes $d$ hier als tenuis erscheint.

§ 69. In den ubrigen, den weitaus meisten făllen, orscheint altes $\hat{o}$ als $\mathscr{e} a$. Dor entsprechende umlaut ist $\hat{e}$. Beispiele: faear (fuora), št aal (stuol), bleam (bluoma), daan (tuon), baeaf (*buobo), ceavr (got. *ôfrs), braeadr (bruoder), blacat (bluot), baeazm (buosam); umlaut: fickln (fuolen), fầrn (fuoren), drếl (truobi), blêi $($ bluot $=$ blite), fêtdr (/uodar).

Anm. In bracaln ist $a^{2} a$ wol unter einfluss des $l$ entstanden, nachdem $\hat{c}$ verkürzt worden (vgl. $\$ 36.43$ ).

$\S 70$. Die verbindung -ôn- zeigt sich als $a a,-\hat{o} j$ - als $\hat{e}$ : raea (ruowa); blân (bluojan), frôe (fruoi).

5. Westgerm. $\hat{\imath}$.

$\S 71$. Westgerm. $\hat{u}$ erscheint als $\alpha^{2} o^{2}$ vor $k, g>x$, z, vor $n>n$, endlich vor $d>g d$. Der umlaut ist $e^{2}$ vor $k, g>$ $\chi, j$; $e$ vor $n>n$ und vor $d>d d$. Beispiele:

1. $b \varkappa^{2} o^{2} x$ (bûh), br $x^{2} o^{2} x n$ (brâchen), $d e^{2} o^{2} x$ (mhd. $d \hat{u g e}$ ), 
$z e^{2} o^{2} z^{n}$ (sagan). Umlaut: $b e^{2} \chi n$ (*tachên, mhd. bụchen), ge $e^{2} \chi$ (ndd. juche).

2. $b r c^{2} o^{2} x(b r \hat{u} n), t s a^{2} o^{2} x(z \hat{u} n), l e^{2} o^{2} x$ (mbd. alun); u mlaut: tsenian (zäunen).

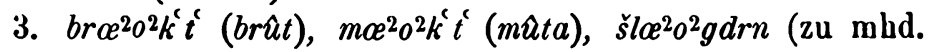
sluder), š $\aleph^{2} o^{2} g d r n$ (ndd. schudderen); umlaut: ed'dr (atiro), gak'ret tsl (krât: gekratitsel).

$\S 72$. Altes $\hat{a}$ wird zu $o^{2}$ vor $m$. Der umlaut ist $e^{2}: d o^{2-}$

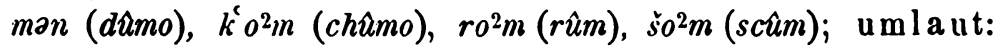
$d e^{2}$ man (pl. von dûmo). (scûvala).

A n m. 1. $o^{2}$ zeigt sich anch in $r o^{2} p(r u p a), z o^{2} f n(s u ̂ f a n), \quad \check{s} o^{2} f$

A n m. 2. Wegen $b e^{2} l(b \hat{u} l i a), f e^{2} l\left(f \hat{u} l\right.$ ), $m e^{2} l$ (mùla) ist verkürzung des $\hat{a}$ zu $o^{2}$ auch vor $l$ anzusetzen (vgl. $\$ 64$ ).

$\S 73$. In den ubrigen, den meisten fällen, ist altes $\hat{u} \mathrm{zu}$ $\varkappa o$ geworden. Der umlaut zu diesem $\propto o$ ist $e^{2} e$. Beispiele: zceor (sûr), badceorn (mhd. tûren), hoof (hûba), dceof (tuba), brœos (bruss), t'oozni (tusunt); u mlaut: fe er (fâir), he $e^{2} e f k n$ (häubchen), he ezr (pl. von hos).

§ 74. Die verbindung - $\hat{u}_{w}$ - erscheint nur umgelautet als $e^{2}$ und $\hat{a}, e^{2}$ wahrscheinlich unter einfluss des folgenden $l$ (vgl. § 72 a. 2): $e^{2 l}$ (âvila); trôen (trûwên), bôen (b̂ân).

\section{c) Diphthonge.}

1. Westgerm. ai.

$\S$ 75. Westgerm. ai hat sich regelmässig $\mathrm{zu} \hat{\imath}$ weiterentwickelt: zîl (seil), lîrn (lêren), hîm (heim), rîn (reini), his

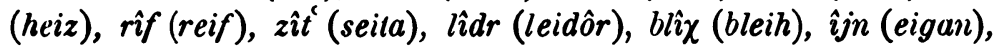
hizr (heisi), gist' (geist).

Anm. In einigen fällen - ich habe meist beispiele vor $l, m, n-$ tritt verkurzung des $l$ zu $e^{9}$, sehr selten zu $\imath^{7}$ und $a^{2}$ ein. Bedingt ist diese verktirzung durch das antreten von bildungssilben. Beispiele: hîl - he $e^{2} i^{2} \chi$ (heilag); hîm - he $e^{2} m l^{2} \chi$ (mhd. heimelich), $k^{2}$ lìn - comp. k'le $e^{2} n r$ (kleini); zîl - pl. ze $e^{2} l r(s e i l)$, bîn - pl. be $e^{2} n r$ (bein); tsvin -

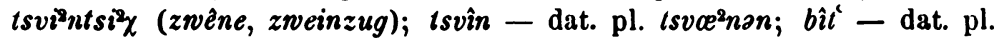
$b e^{2} d n$ (beide).

§ 76. Die gruppen -ain- und -aih- erscheinen als $\hat{\imath}$, aijals $0^{2} \jmath^{2}$. Beispiele: a) $\hat{\imath}$ (êwa), $\hat{\imath} v i^{2} \chi$ (êvig), šnî (snêo), zîl (sêla), $t \sin (z e ̂ h a) .-$ b) $o^{2} \jmath^{2}(e i), \quad l^{2} \partial^{2}$ (as. leia), $m o^{2} \jmath^{2}$ (meio), mo2orar (meior).

A nm. Das neutram der zweizahl lautet im dialekt tsvi2a. 
2. Westgerm. au.

$\S 77$. Westgerm. au erscheint als $a$ und $y u$, und zwar entspricht $\hat{u}$ dem ahd. $a u, o u$, dagegen $y u$ dem monophthongierten ahd. $\hat{o}$. Der umlaut von beiden stufen ist $\hat{\imath}$. Beispiele:

1. rum (mhd. roum), zûm (soum), lufn (louffen), gluvn (giloubo), rûx (rouh), ûx (ouga); u mlaut: bîm (pl. v. boum), štrîfn (*stroufen); glîvn (gilouben), bîjn (bougen).

2. gryus (grôz), blyus (blôz), nyut (nôt), ryut (rôt), dyut (tôd), byun (bôna), lyun (lôn), lyus (lôs), ryust (rôst), yur (ôra), ryur (rôr), hyu (hôh), flyu (flôh), šyun (scôno); u mlaut: hîrn (hôren), šîn (scôni), nîdi $\chi$ (nötig), grîs (grösse), lizn (lôsen), gakiris (chrôsi).

$\mathrm{Anm}$. $\hat{\imath}$ wird zu $e^{2}$ verkürzt: gryus $-g r e^{2} s r$ comp. (grôz), hyu

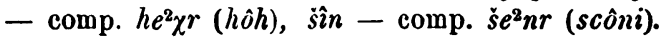

$\S 78$. Die verbindung -oun- erscheint stets umgelautet als $\hat{x}: \hat{e}$ (ouwa), frôe (frouwa), hêen (houwan), ḱrôn (chrouwôn), dồn (douven).

3. Westgerm. iu.

$\S 79$. Westgerm. iu entwickelt sich zu $e$ vor altem $d, n$ $>d^{\prime} d, n$ (vgl. §63. §71, 2. 3): bed'dn (biotan), het't (hiuto), ded'dn (diuten), let't (liuti); neń (niun).

$\S 80$. Altes $i u$ wird zu $e^{2}$ vor $t, p>s, f$; ferner vor $k, g>\chi$, wenn nicht ein 'brechung' bewirkender vocal folgte. Beispiele: 1. fle $2 s n$ (fliozzan), ge 2 sn (giozzan), špe ${ }^{2} s$ (spioz); št $^{2} e^{2} f$ - (mhd. stief-), de $e^{2} f\left(\right.$ tiof); - 2. tse $e^{2} \chi$ (zing).

$\S 81$. Vor $k, g>\chi, j$ wird altes $i u$ zu $a^{2}$ : raxn (riohhan),

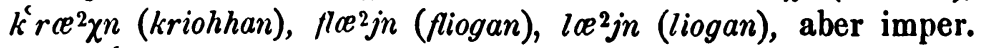
$r e^{2} \chi$ ! kr $r e^{2} \chi$ ! fle $\chi$ ! le $e^{2} \chi$ ! (vgl. § 80, 2).

$\S 82$. In den ubrigen fällen erscheint altes $i u$ als $\hat{a}$. Bei-

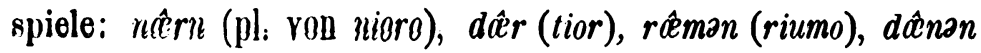

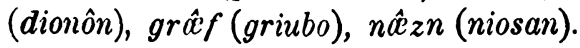

A n m. 1. Neben rểman (riumo) auch $r e^{2} m a n$; doch hat rêman die speciellere bedeutung 'ledergürtel'.

A n m. 2. Als $e^{2} e$ erscheint altes $i u$ in gahe $e^{2} e r$ (-hiuri), he $e^{2} e r$ (hiuro), $\dot{s}^{2} e r$ (sciura); ferner vor altem $s$ in der II. III. pers. ind. praes. und im imper. der verben der II. ablautsreihe, vgl. § 137, a. Also auch hier ist, wie $\S 80$, zwischen gebrochenem und ungebrochenem iu zu unterscheiden.

$\S 83$. Die gruppe -iuh- erscheint als $\hat{x}$, -iun-als $-0^{2} 0^{2}$.

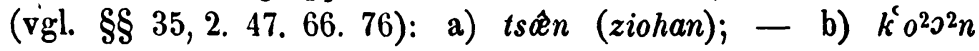


(chiuwan), $k l o^{2} \jmath^{2}$ (chliuwa), $n o^{2} \jmath^{2}$ (niuwi), $r o^{2} \jmath^{2}$ (riuwa), tro $0^{2}$ (triuwa und triuwi).

\section{Die westgermanischen balbvocale.}

1. Westgerm. $w$.

\$ 84. Westgerm. $w$ ist geschwunden in den gruppen [ $w l-$, ] wr-; ferner nach vocalen; nach $l, r$ nur, wenn es nicht ursprunglich durch folgenden vocal geschutzt war. Sonst ist altes $w$ zu $v$ geworden, welches als $f$ auslautet.

a) $w$ geschwunden: 1. re $e^{2} e s n$ (as. wrîtan), ra ${ }^{2} s t$ (ags. wrist),

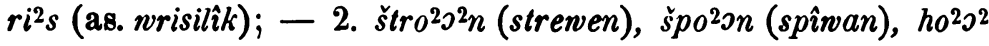

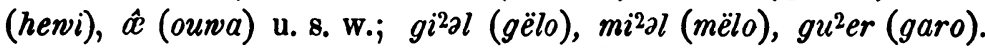

b) $n$ erbalten: 1. vôr (wâr), ḱviªln (quellen), švi2ari (swërt), tsve2lf (zwelif), u. s. w.; - 2. ge 2 lvn (gelb machen), švalf (swalawa), ga'rvn (gariwen), narf (narwa), farf (farawa).

$A \mathrm{~nm}$. 1. Geschwunden ist $w$ auch in $z a^{2} s i^{\prime} r$ (swëster) und $t^{\prime} a^{2} \dot{s} n$

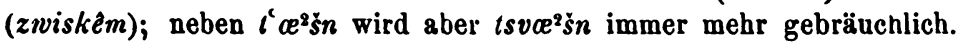

A n m. 2. $\quad w$ ist erhalten in $\hat{i} v i^{2} \chi$ (enîg).

A $\mathrm{nm}$. 3. In $a^{2} r b a s$ (araweiz) ist übergang von $w \mathrm{zu} b$ vorhanden; wenn brañen buchstäblich ags. wringan ist, wäre auch hier übergang von $w$ zu $b$ zu constatieren (vgl. Wolff, Konsonantismus 8. 40).

2. Westgerm. $j$.

§ 85. Westgerm. $j$ ist nur noch im anlaut vorhanden, wo es als $g$ erscheint. Wenn nebenher auch formen mit $j$ gehen, so sind diese doch sicher neueren ursprungs, da die mit $g$ anlautenden stets eine speciellere bedeutung haben. Ueber $j$ in anderen stellungen im wort vgl. $\$ \S 61.66 .70 .76$. Beispiele:

guªzn (jagôn) 'vertreiben' - juzazn allg. 'jagen'; gônurn (jamarôn) 'naschhaft begehren' - jômrn 'jammern'; gre ${ }^{2} a$ (jung) 'knabe' - jaw 'jung'; gôr, jîr (jâr); ge $\chi$ (ndd. juche), $g e^{2} n r$ (jënêr), gat $k^{\prime} n$ (jucchen), gańln (junge bekommen), gônan $(j a n)$.

A $\mathrm{n}$ m. Die bejahungspartikel lautet $\chi a$ !, in nachlässiger rede $h e^{2} a$ !

III. Die westgermanischen liquiden und nasalen.

1. Westgerm. $l$.

§ 86. Westgerm. $l$ ist uberall, auch in ursprunglicher gemination, als $l$ erbalten. Merkwürdiger weise erscheint es 
nie mouilliert, wie ich es in angrenzenden mundarten wol gehört habe (vgl. Wolff, Konsonantismus s. 17, anm. 1).

2. Westgerm. $r$.

§ 87. Westgerm. $r$ ist in allen stellungen als $r$ erhalten.

Anm. 1. Auslautendes $r$ fällt ab in den einsilbigen $h i^{2} a$ und $a$ (ër), vî (wër), dî̀ (dër), dô (dâr), vo (wâr), hôe (hiar), mî (mêr), vgl. Wolff a. a. o. 21. Dagegen $d u^{2} \partial r$ (dara), vuter (wara) dahin, wohin?

A $\mathbf{n}$. 2. In $v i^{2} l^{\prime}$ (wirdit) bat assimilation des $r$ an $t$ stattgefunden, ebenso in $v e^{2 t} t, v e^{2} d n$ ( $v u r d i$ etc.). Häufig wird nach analogie des infinitivs $v e^{2} r d n$ das $r$ widerhergestellt: $v i^{2} r t^{2}, v e^{2} r t$.

3. Westgerm. $m$.

$\S 88$. Westgerm. $m$ ist uberall und stets als $m$ erhalten, ausgenommen natürlich die $\mathrm{zu} n$ gewordenen ungeschutzten alten flexions-m.

Anm. 1. Mit rücksicht auf das nhd. wichtig ist die erhaltung des $m$ in fadm (fadam), bceazm (buosam), bìssm (bésamo) und : ihnlichen.

A n m. 2. Dem $u$ in folgender silbe assimiliert hat sich $m$ in $k^{2} u^{2} n$ (choman), nizon (nëman). Mit folgendem $g$ hat sich $m \mathrm{zu} v$ verbunden in boe ${ }^{2} a m r l^{\prime}$ (mhd. boumgarte).

4. Westgerm. $n$ [und $n]$.

$\S 89$. Westgerm. $n$ ist gewöhnlich als $n$ erhalten, die gemination $n n$ vereinfacht; ebenso erhalten ist westgerm. $x$ in den gruppen $n g$ und $n k$.

§ 90. Durchkreuzt ist obige regel zuerst durch das gesetz der palatalisierung, welchem $n$ und $x$ unterliegen, und das gesetz der gutturalisierung, dem in gewissen făllen $n$ unterworfen ist.

1. Einfaches $n$ wird mouilliert durch vorhergehendes altes $\hat{\imath}$ oder $i u$ und erscheint als $\dot{n}$, in der verbindung $n p$ und $n d$ durch vorhergehendes $i$ oler jeden palatalisierten (umgelauteten) vocal (vgl. §63. § 79. § 45. § 29, 3. § 57). Beispiele: a) šeñan (scînan), šveń (swîn), grenian (grînan), leńda $a x$ (lîntuoh), veń ( în), feń (fîn); neń (niun); - b) fańdn (findan), gašvañ ' (*swirdo), gazañt' (gisindi), rańt (rind), k’ańt (chind); bańdn (bintan), lańt (linta), rańt (rinta), vańt (wint), grañi (grint), zœeńt (mld. sint); vańt r (vintar); - c) ón't (enti), bə hon't (bihenti), lońdn (lenti, pl.), šondn (scenten); zañt (sunta).

2. $\mathrm{Zu} \otimes$ gutturalisiert wird einfaches $n$ nach altem $\hat{u}$, und in den gruppen -unp-und -und- (vgl. \$§ 71. 57): $b r c^{2} o^{2} z$ (bran), 


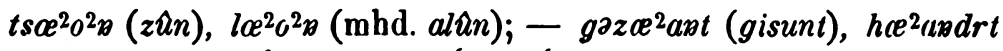

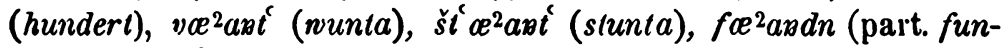
dan), forast (pfunt).

3. $n$ (altes, und erst in der mundart entwickeltes) wird, ausser in der gruppe $x k$, vgl. $\S 42,2$. $\S 31,3$. $\S 57,3$, durch jeden vorhergehenden palatalen oder palatalisierten vocal mouilliert und erscheint als $\dot{n}$ (vgl. § 45. § 31. § 71. § 57, 2). Beispiele: a) drañan (dringan), fańr (fingar), galañan (gilingan), brañan (ringan), zañan (singan); - b) droñan (drengen), oń (engi), mońan (mengen), zońan (mhd. sengen); - c) tseńan (zäu-

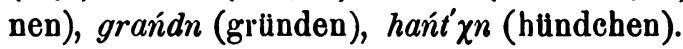

Anm. Die zwischen vocalen sehr merkliche dehnung des $n$ rührt ohne zweifel davon her, dass altes $\hat{\imath}$ oder $i u$, welche kurz erscheinen einen teil ihrer quantität an das folgende $n$ abgegeben haben. Ebenso erklärlich ist die länge des aus $\boldsymbol{x}$ entstandenen $\tilde{n}$, vgl. $§ 2$.

$\S 91$. Altes $n$ vor $s$ und $f$ ist stets geschwunden; der vorhergehende vocal erscheint gedehnt: $g 0^{2} \jmath^{2} s$ (gans), $l 0^{2} \jmath^{2} s$ (linsi), $d 0^{2} 0^{2} z n$ (dinsan), $o^{2} 0^{2} s l t^{t}$ (unslit), aeas (uns); - ro2oft (mhd. ranft), fo $0^{2} \rho^{2} f$ (finf), farnoeaft (firnunft); - $t s^{2} \jmath^{2} s$ (zins).

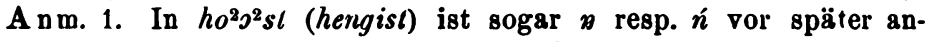
getretenem $s t$ ausgefallen. Dasselbe ist der fall in $f o^{2} s^{2} \sin \left({ }^{*} p f i n g u s(i n)\right.$.

A n m. 2. In zents (ségansa) hat sich $n$ vor $s$ erhalten (weil es ursprünglich in nebentoniger silbe stand?); $-a^{2} m z o^{2} n t s t^{t}$ ist spätere nachbildung des hd. umsonst; die ältere form lautet $a^{2} m z c^{2} s t^{t}$ (whd. umbe sus).

§ 92. Ueber den wegfall von auslautendem flexions-n im zusammenhang der rede siehe $\S 9$.

Anm. 1. Das $n$ der schwachen declination geht in rêxl (rëcho) und spadl ( ${ }^{*}$ sputo) in $l$ über; ebenso das $n$ in $z u^{2} m l n=$ samanôn.

A n m. 2. In brôn (brinnan und brennen) hat sich $n n$ dem flexions-n

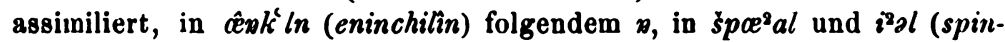
nala und elina) folgendem resp. vorhergehendem $l$, in grceaman (mhd. gruonmat) folgendem $m$.

\section{Die westgermanischen spiranten.}

1. Westgerm. $s$.

$\S 93$. Westgerm. $s$ erscheint anlautend vor vocalen und inlautend zwischen sonoren als $z$, auslautend als $s$. Das aus $h s$ entstandene $s$ bleibt stets stimmlos. Altes $s$ in den anlautenden verbindungen $s l, s m, s n, s w, s p$ und $s t$, ferner $s$ nach $r$ gehen in $\breve{s}$ uber; $\breve{s}$ nach $r$ wird, wenn sonor folgt, stimm- 
haft zu $\check{z}$. Altes $s k$ erscheint stets als $\check{s}$, ohne je stimmbaft zu werden, ausser im auslaut vor anlautendem vocal (vgl. § 4). Altes $s s$ wird zu $s$ vereinfacht. Beispiele:

1. zôn (sagên), zên (sâjan), füazln (fasôn), fäaldzn (feliso), do $0^{2} \partial^{2} z n$ (dinsan).

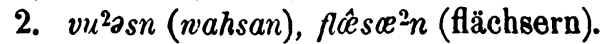

3. šlôn (slahan), šme $e^{2} e s n$ (smîzzan), šned dn (snîdan), šve $e^{2}$ jn (swîgên), špuªrn (sparôn), št ôn (stân). Dagegen: re $e^{2} e s p r n$ (mhd. riuspern), fastn (fastên).

4. hîrš (hirsi). - $\hat{x} r z ̌ l i{ }^{2} \chi$ (ärschlings), mîržl (morsâri).

5. šred dn (scrîtan), šyun (scôno), hî̌šn (eiscôn).

6. gav $\hat{e}^{2} s,-s-($ givis, $-s s-), r u{ }^{2} a s,-s-(r o s,-s s-)$.

A n m. 1. Vereinzelt erscheint $s$ zwischen sonoren stimmlos: $b i^{2} \partial s m$ (bësamo), gîsl (geisala), hasl (hasala), o os sitt (unslit).

A n m. 2. rašpl (raspel) und ḱaśpâri (Caspari) stehen sicher nnter obd. einfluss; neben ḱa ašpâri auch der eigenname ḱkaspr.

§ 94. In fremdwörtern verwandelt der dialekt anlautendes stimmloses $s$ häufig in $t s$, vgl. Wolff, Konsonantismus, s. 52. Beispiele: tsaloºt (mbd. salât), tsalm (Psalm), tso2or (Sarah) etc. etc.

A n m. 'Sächsisch' lautet im 'sächsischen' dialekt sak' tsoš, häufig auch tsak' tsoś. Aus anlautendem $s$ und ts ist mit sicherheit zu schliessen, dass der name von Oberdeutschen iiberkommen worden. 'Sächsisch' müsste eigentlich *zêesoš oder *zizosoš lauten.

2. Westgerm. $f$.

$\S 95$. Westgerm. $f$ erscheint an- und auslautend als $f$, inlautend zwischen sonoren als $v$, sonst auch als $f$. Beispiele: 1. foosi (fust), fle es (fliz), fryu (frô). - 2. grêf, -v- (grâo) ceavr (got. *ôfrs), tsve $e^{2} e v l$ (got. tweifls), haenf, -v- (as. hôf),

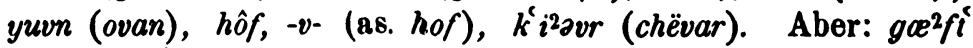
(gift), laft (luft):

Anm. 1. Obwol von sonoren umgeben, erscheint altes $f$ stimmlos in $t^{2} e^{2} r f n$ (durfan), gafl (gabala), śo $o^{2} l$ (scuvala).

A $\mathrm{nm}$. 2. Altes $f$ ist ausgefallen in hî̉ (mhd. hovesch).

3. Westgerm. $p$.

$\S 96$. Westgerm.. $p$ ist stets $\mathrm{zu} d$, auslautend $\mathrm{zu} t^{c}, b b$ zu $l$ verschoben worden. Jenes neue $d$ steht mit dem alten unter dem einfluss derselben mouillierung durch vorhergehendes $\hat{\imath}[i u]$ oder nachfolgendes $\chi$ (vgl. $\S 103$ ). Beispiel: $1 . p p>t$ : lốtn (letto, isl. lepja), môt (ags. mopbe), šma ${ }^{2} \hat{t}$ (ags. smibpe); 
špôt (got. *spuppôn, Kluge Etym. wb. 324). - 2. ḱ et't (chîdi), led dn (lîdan), vet't (nîda), šned'dn (snîd(ın), net' $t^{\prime}$ (nîd).

A n m. Einige anlautende $P$ erscheinen im dialekt als $t: t^{c} u^{2} \partial s t^{c}$

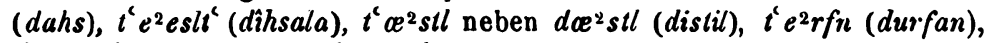
$t^{\prime} \alpha^{2} a n k^{\prime} n$ (dunchôn), t'œozni (as. thûsind), tre esi' ${ }^{2} \chi$ (drîzug). - In $\operatorname{tre}^{2} e s i^{2} \chi$ ist $t$ wol unter einfluss des ihm meist vorangehenden ent (und) bei zusammengesetzten zahlen entstanden. Also aus $d r 0^{2} \partial^{2} a n t^{i}-d r e^{2} e s i^{2} \chi$ - dro $0^{2} g^{2} a n d r e^{2} e s i^{2} \chi$ - $t r e^{2} e s i \chi$. Ebenso $t$ in $t^{\prime} a a(d u)$ unter einfluss des vorangehenden flexions-s der II. pers. Daneben aber auch doea, unbetont $t^{\prime} s, d o$.

\section{Westgerm. $h$.}

$\S 97$. Westgerm. $h$ ist uberall geschwunden, ausser im anlaut mit folgenden vocal, in der verdopplung und der verbindung $h t$. Im anlaut erscheint es als $h$, in der gemination und der gruppe ht als $x$ oder $\chi$, je nachdem der voraufgehende vocal guttural oder palatal ist. Beispiele:

a) $h$ geschwunden: 1. ladn (got. hlajan), rań (as. hring), nijn (as. hnêgan), ve $e^{2} s$ (as. hnît). - 2. $h$ zwischen vocalen, vgl. § 35. § 41. §61. §66. § 76. § 83. - 3. t $u^{2} \partial s t$ (dahs),

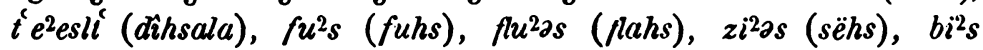
(buhsa). - 4. mu2r (morha), für (furuh), zu'al (salaha), bəfi2aln (bifëlhan).

b) $h$ erbalten: 1. hi2avn (heffen), hoe ${ }^{2} r t s$ (hërza) u. 8. w. -

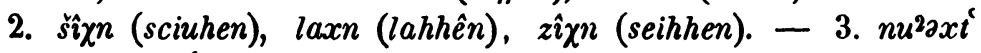
(naht), u'axt (ahta), vu2 $u^{2} x t l$ (vahtala).

An m. 1. Vereinzelt hat sich altes $h$ noch erhalten in: $h e^{2} \chi r$, comp.

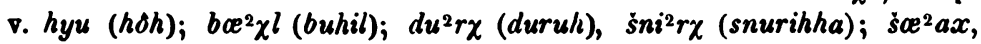
-z- (as. scoh) scheint auf grammat. wechsel zu beruhen.

A n m. 2. $h o^{2} k^{t} t s o t^{c}$ (hochzeit) ist sicher lehnwort, obwol allgemein gebräuchlich; dafür spricht ausser dem $k$ besonders die qualität des vocals.

V. Die westgermanischen verschlusslaute.

a) Die tenues.

1. Westgerm. $p$.

$\S$ 98. Westgerm. $p$ erscheint regelmåssig als $f$ nach vocalen und, soweit sich absehen lässt, nach $r$ und $l$. Im anlaut, nach $m$ und in der gemination ist das $p$ in alten dialektwörtern unverschoben geblieben. Wo sich in diesen stellungen ein verschobener laut findet, weist or auf moderne entlehnung. Von einigen wörtern kommen doppelformen mit und obne 
verschiebung neben einander vor; daun haben in der regel die formen mit unverschobenem $p$ eine speciellere bedeutung und lassen mich hierdurch ein höheres alter erschliessen. Beispiele:

1. gre $e^{2} e f n$ (got. greipan), lûfn (got. hlaupan), šlôfn (got. slêpan), de $e^{2}$ (got. diups), zîf (seifa, ags. sâpe), haf (got. hups); - do ${ }^{2} r f$ (got. paurp), sarf (ags. scarp), vaerfn (got. wairpan); $h e^{2} a l f n$ (got. hilpan; dagegen z. b. št $a^{2} a l p n=$ nhd. stülpen).

A n m. 1. Für $d a^{2} a l p n$ 'beschwichtigen', besonders vom feuer gebraucht (vgl. Haltrich, Plan zu vorarbeiten für ein idiotikon 8. 9), ša ${ }^{2}$ alpn 'erdscholle' weiss ich kein etymon.

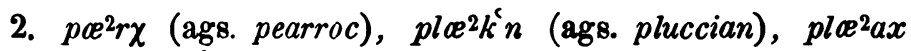
(ags. plôg); paxt (mhd. pfahte), pô (pfâwo), paé2dm (pfédemo), pi2arš (mhd. pfërsich), pi2l (pfuliwi), pae ${ }^{2} p t s$ (pfifiz); dagegen mit verschiebung: fli2ajn (as. plëgan); faf (pfaffo), fo'sont ( $p f a n t)$, $f o^{2} n$ (pfanna), far (pfarrâri), fe $e^{2 l r}$ (pfìlari), fe $e^{2 n a z k}$ (pfenning),

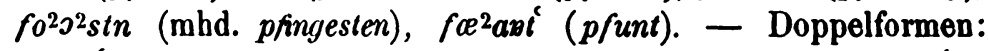
fu'2ast ( $p$ fosto) 'pfosten' im allgemeinen, dagegen demin. pi2asi $\chi n$ 'setzling der pflanzen'; flo2onts (pflanza) 'pflanze' im allgemeinen, dagegen plo2onts 'junge gartenpflanze, noch bevor sie in's freie versetzt ist'; $p i^{2} p$ ( $p f \hat{\imath} f a$ ) 'pfeife, weinpipe', $f e^{2} e f$ 'tabakspfeife' ('tabak rauchen' heisst dagegen stets $p i^{2} p n$, nie $f e^{2} e f n$ ).

3. o $^{2}$ mprt (ampfaro), zamp (mhd. sumpf), klampn (ndd. klump), št ampn (stampfôn), št ampi ${ }^{2} \chi$ (stumpf), štrump (mhd. strumpf), di ${ }^{2} m p i^{2} \chi$ (mhd. dimpfen, dampf, dempfen); dagegen aus dem nhd. entlehnt k'o2ompf (ags. camp), k'ro2ompf (as. kramp).

4. apl (apful), tsôp (zopf), šcepn (scepfen), ḱlôpn (chlopfôn), k'nup (knopf), gegen k'nîtl (nhbd. knouf) etc. etc.

A nm. 2. Verschoben ist $p p$ im lehnwort ksafr (kupfar).

2. Westgerm. $t$.

$\$ 99$. Westgerm. $t$ ist nach vocalen (ausser in der gruppe tr) stets zu $s$ verschoben. Eine ausnahme machen nur die aus einem grossen teil des fränkischen bekannten $d e^{2} t, d e^{2}$ in (diz), dat', $d 0^{2} \partial t^{t}, d a t^{\prime}(d a z)$, at (iz), alnt (allez) und das neutrumbildende endungs- $t(-z)$ bei den adjectiven. Altes $t$ im anlaut (ausser in der verbindung $t r$ ), nach nichtvocalen (ausgenommen das $t$ in $f t, s t, h t$ ) hat sich zu ts entwickelt, ebenso auch in der gemination. 
Da die verhältnisse hier so einfach daliegen und in einem grossen teil des fränkischen sprachgebiets dieselben sind, ist die anfubrung von beispielen wol nicht nötig.

Anm. 1. Eine ausnahme macht das in Mediasch selbst freilich

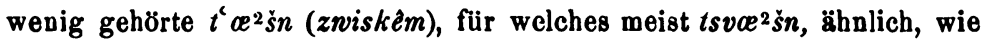
für alnt unter einfluss des nhd. meist alos gebraucht wird.

A nm. 2. Von einzelheiten wäre anzufuibren, dass ahd. latla, ladda (Kluge, Etym. wb. 194) im dialekt lats, und ratta rats lautet.

A $\mathrm{n}$. 3. Aus $t$ entstandenes $s$ wird ausnahmsweise stimmhaft in

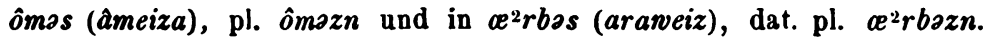
Stimmhaft ist $t s$ geworden in gundzr (ganazzo).

3. Westgerm. $k$.

$\S 100$. Westgerm. $k$ ist nach gutturalen vocalen zu $x$, nach palatalen und palatalisierten zu $\chi$ verschoben worden. In allen ubrigen stellungen erscheint es als $k^{c}$ (vgl. § 24).

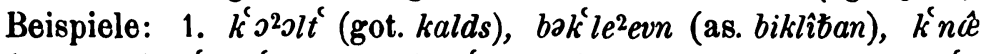

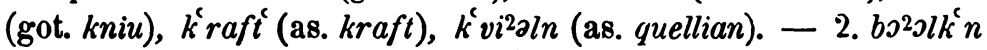
(balko), bi ${ }^{2} r k$ ' (ags. beorc), diªk n (got. pagkjan). - 3. ak'as (as. accus), dôk (toccha), hak'n (ags. haccian).

A n m. 1. $k$ nach $n$ schwindet vor $t$ mit dehnung des $x$ wie es scheint, zum mindesten liegt auf demselben ein grösser expirationsdruck; z. b. $d i^{2} x t^{\prime}$ 'denkt'.

An m. 2. In einigen nominibus auf $x$ (ans altem $k$ ) wird dieses $x$, wenn es durch die flexion inlautend wird, zu $z, j$, also stimmbaft. Diese erscheinung ist sicher aus der einwirkung der nomina auf $x$ aus ursprünglichem $g$ zu erklären. Beispiele: $d^{2} \supset x(d a h)$, pl. dâjj; $b a^{2} a x$

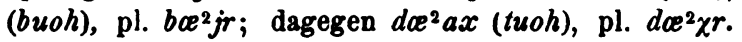

\section{b) Die medien.}

1. Westgerm. $b$.

$\S 101$. Westgerm. $b$ zeigt sich im anlaut stets als $b$. Nach sonoren erscheint altes $b$ stets als $v$, auslautend und vor stimmlosen lauten als $f$. Die geminata $b b$ findet sich als $p$ im dialekt. Beispiele:

1. be ${ }^{2} e s n$ (got. beitan), brêxn (got. brikan), blôzn (got.

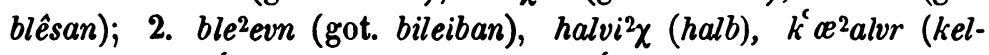
bir); - 3. št af, -v- (stoup-, -b-), hift' (got. haubib), pl. hîvdr; 4. ḱrae ${ }^{2} p$ (chrippa), rae ${ }^{2} p$ (rippa), re $e^{2} p$ (ruoba, *ruobja), ro ${ }^{2} p$ (rappa).

Anm. 1. $b$ nach $r$ hat sich als $b$ erhalten in arbat (got. arbaips).

Anm. 2. Westgerm. $m b$ ist uberall zu $m$ verschmolzen. Auslautendes $b$ hat sich dem $m$ assimiliert, ohne dass dies an der quantität 
des $m$ bemerkbar wäre: ḱ $\rho^{2} o m$ (chamb), loºm (lamb), tum (tumb). In innern des wortes hat sich $b$ vorangehendem $m$ assimiliert in šce $m l$ (*scimbal). In compositis: înı (einbar), brômr (brâmberi). Als $p$ erscheint jedoch $b$ im anlaut des zwciten teils in compositis, wenn der erste auf $t$ ausgieng: ha'mpr (hintberi), $a^{2} r p l$ (ërtberi).

A nm. 3. Ohne spuren $z u$ hinterlassen ist $b$ geschwanden in $h u^{2} n$ (habên) und gi $i^{2} a n$ (gëban); der imperat. lautet haf, gae $f$.

2. Westgerm. $d$.

$\S 102$. Westgerm. $d$ erscheint im dialekt in allen stellungen als $d$. Auslautend wird es zu $t$. Die geminata $d d$ zeigt sich als $t$. Beispiele: für $d d>t$ : $d r a^{2} t^{t}$ (ags. pridda), $m c^{2} t n t^{t}$ (as. middia). Dagegen $b i^{2} d n$ (ags. biddan) mit $d$, vielleicht unter einfluss des participiums $g^{2} b c e^{2} d n$.

$\S 103$. Wie bei $n$ (vgl. $\S 90)$ wird die allg. regel auch hier durchkreuzt vom gesetz der palatalisierung und der gutturalisierung.

1. Nach altem $\hat{\imath}$ und $i u$ erscheint $d$ als $d^{\prime} d$, auslautend als $t^{\prime} t$. Vor $j$ zeigt sich $d$ als $d^{\prime}$, vor $\chi$ als $t^{\prime}:$ red $d n$ (rîtan),

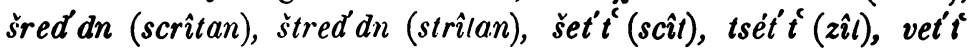
(nît), k'ret i (krîda), zet't (sida); - bed'dn (biotan), ded'dn (diotan), het't (hiuto), let't (liuti); - bli'2at' $\chi^{\prime}$ (dem. v. blat), gald' jn (galgo).

2. Nach altem $\hat{u}$ entwickelt sich vor $d$ ein $g$; lautete es als $i$ aus, ein $k^{k}$. Wird das alte $\hat{u}\left(>\boldsymbol{a}^{2} o^{2}\right)$ umgelautet zu $e$, so erscheint $d$ wie nach altem $\hat{\imath}$, iu als $d^{\prime} d$ resp. $t^{\prime} t$. Bei-

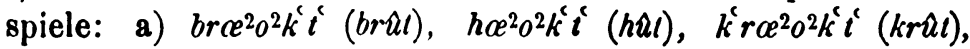
$m a^{2} o^{2} k^{\prime} t^{\prime}$ (mûlu), šl $a^{2} o^{2} g d r n$ (zu mud. sluder), š $a^{2} o^{2} g d r n$ (ndd. schudderen); - b) ed'dr (âtiro), gak'ret'tsl ('gekräutsel').

A n m. In $b e^{2} g l$ (bùtil), $t s e^{2} k^{\prime}\left(i^{2} \chi\right.$ ('zeitlich' = häufig), $n e^{2} k^{\prime} l i^{2} \chi \quad(z u$ niol, 'rasch, hurtig') ist $d^{\prime} d-t$ ' vor $l$ in $g-k$ ' ubergegangen.

$\S 104$. Als weniger durchgreifende, zum teil 'unorganische' erscheinungen sind anzufuhren:

1. Die gruppe $d r$, welche in dialekt stets hätte erhalten bleiben sollen, scheint anlautend von der gruppe $\operatorname{tr}$ beeinflusst zu sein; inlautend scheint gegenseitige beeinflussung stattgefunden $\mathrm{zu}$ haben. Beispicle: a) trêfn (ags. drepan), trôpn (as. dropo), træoori ${ }^{2} \chi$ (ags. dreórig), tri2avr (ags. Urabbe, hefe), trutî (dän. drude); b) nôt $r$ (as. nâdra), dagegen blôdr (as. blâara), rei'ir (ags. hrîder), hî̀ (ags. hlếder); füair (as. fudar)

Beiträge zur geschichte der deutschen sprache. XII. 
und motir (as. môdar) sind auch hierher zu ziehen; c) k'adr (got. *katra, Kluge, Etym. wh. 155), tse 2 drn (an. titra).

Anm. Zu den beiden letzten punkten vgl. Sievers, Beiträge $V$, 522. - Erhalten sind $d r$ resp. $t r$ in facadr (ags. fồor), voe ${ }^{2} d r$ (ags.

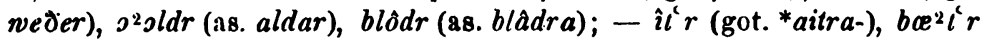
(got. baitrs).

2. Einige als $t$ auslautende alte $d$ erscheinen auch inlautend als $t$ : nôt (ahd. nât), zôt (as. sâd), drôt (ags. prêed), - hît (got. hairdeis), hae ${ }^{2}$ t $^{c}$ (got. hairda), vyurí (got. waurd), byurtn (borto), gueartn (as. gardo).

3. Anlautendes $d$ erscheint als $t$ in fart $e^{2} l d^{\prime} j n$ (as. fardiligôn), ituavn (ags. dofian), têedi'jn (mhd. tagedingen), it $e^{2} e v l$ und de evl (diabolus).

4. Ein 'unorganisches' $t$, welches inlautend zu $d$ wird, entwickelt sich in einigen wörtern nach auslautendem dauer-

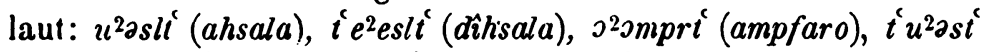

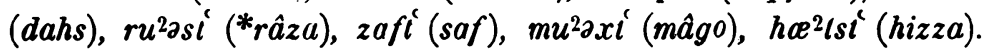

5. Ein ebenfalls 'unorganischer' $t$-laut entwickelt sich zwischen $l, m, n, \dot{n}, f, p,[k]$ einerseits und $s, \dot{s}, \chi, z[\dot{z}], j$ andererseits. Vor $s, \check{s}$ ist es ein $t$, vor $z[\check{z}]$ ein $d$, vor $\chi, j$ ein $t$ resp. $d^{\prime}$. Beispiele: facaldzn (feliso), fós oltš (falsc), $b^{2}$ olt' $\chi$ (balg), gald'jn (galgo); - tse ${ }^{2} m t s$ (dän. timss), le $e^{2} m t^{\prime} \chi n$ (lämmchen), hants (Hans), hieńt' $\chi^{n}$ (dem. v. hano), mańtš (men-

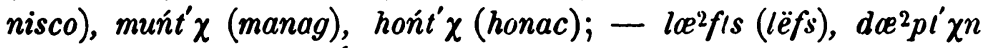

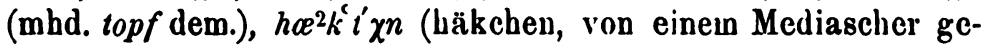
sprochen).

6. Vorhergehendem $n$ assimiliert sich $d$ in šaenan (scintan) und ontsañon (-zunten).

7. $d$ wechselt vor $l$ sebr leicht mit $r$; beispiele: $s^{2} e^{2} d n$ und $s e^{2} r l a n$ (schlitteln), $t s e^{2} d l$ und $t s a^{2} r l$ (mlat. scedula), far-

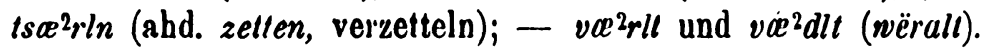

3. Westgerm. $g$.

$\S 105$. Westgerm. $g$ erscheint anlautend stets als $g$, inluutend nach gutturalen vocalen als $\zeta$, nach palatalen, palatalisiciten vocalen und nach $l, r$ als $j . \quad \xi$ und $j$ werden auslautend und vor stimmlosen lauten zu $x$ resp. $\chi$. Westgerm. gg erscheint im dialekt als $k$. Beispiele: 1. gas (gazza), glât

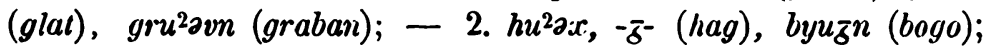

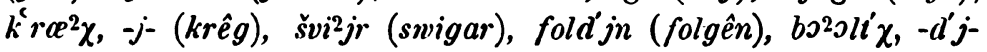




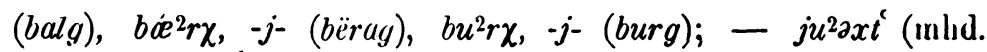
jaget), fo ${ }^{2} l t^{\prime} \chi t^{i}\left(3 . \mathrm{p}\right.$. sing. ind. praes. v. folgên); - 3. $m c^{2} k^{\mathbf{c}}$

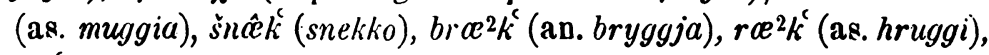
$\hat{c} k^{\prime}$ (as. eggia).

$\S 106$. Westgerm. $n g$ wird in der mundart folgendermassen behandelt. War $n g$ ursprunglich durch folgenden vocal geschutzt, assimilierte sich $g$ dem vorangehenden $x$, worin die zwischen sonoren hervortretende dehnung des $x$ ihren grund hat (vgl. §2). 'Trat $n g$ in den auslaut, verstummte $g \mathrm{zu} k$, sodass $n g$ als $n k^{k}$ erhalten blieb. Ausgleichung hat gewöhnlich vom nominativ oder infinitiv aus stattgefunden. Beispiele:

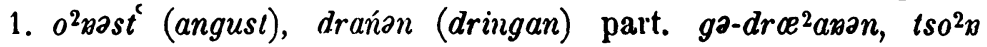

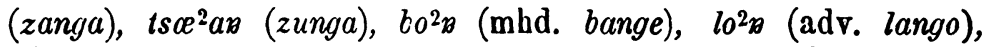

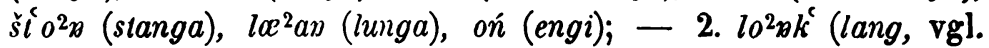
oben $l 0^{2} n$ ), comp. $l c^{2} n k^{i} r$ (dagegen vom adverb $l e^{2} x r$ ); $k^{k} e^{2} n a x k^{c}$ (chuning) und so alle auf -ing; $g 0^{2} x k^{c}$ (imperat. von $g \hat{o} n=g a \hat{n}$ ), praeterit. aber $g e^{2} n$, weil plur. ge $e^{2}$ zan etc. - 3. Von den obliquen casus aus hat ausgleichung stattgefunden in $90^{2} \mathrm{x}$

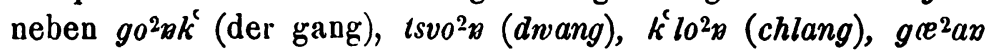
(jung), døeń (ding).

$\S 107$. In folgenden fällen ist $g$ zwischen vocalen gcschwunden, wonach vorhergehender kurzer vocal gedehnt wurde: drôn (tragan), k'lôn (chlagôn), hôl (hagal), ônan (agana), zôn (sagên), lo $0^{2}{ }^{2} n$ (ligen), rên (rëgan), zênts (sëgansa); mêt (magat).

\section{FORMENLEHRE.}

\section{A. Declination der substantiva.}

I. Die masculina.

$\S 108$. Die masculina scheiden sich in der heutigen mundart deutlich noch nur in die beiden hauptklassen der starken und schwachen declination. Die unterschiede innerhalb der starken declination sind dadurch, dass der umlaut durchgängig zum unterscheidungsmerkmal zwischen singular und plural gemacht worden ist, gänzlich verwischt. Dagegen haben sich in der schwachen declination neue kategorien gebildet: dadurch nämlich, dass im singular teils nach dem nominativ ausgeglichen wurde, dessen ahd. -o in dialekt abfallen musste 
(vgl. § 11), teils nach den obliquen casus, sind zwei hauptklassen entstanden. In der einen unterscheidet sich der plural vom siugular durch das hervortreten des charakteristischen $n$, welches dem ganzen singular feblt; in der andern lauten sing. und plur. gleichmässig auf jenes $n$ aus, wobei der umlaut als unterscheidungsmerkmal beider numeri immer weiter um sich greift.

a) Starkes masculinum.

$\S 109$.

Sing.

Plur.

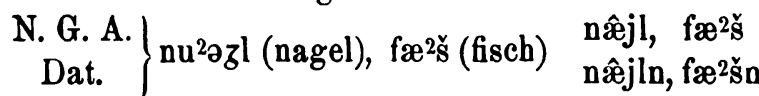

Anm. 1. Die verwantschaftsnamen $f u^{2} \partial t^{i} r$ (fater) und brceadr (bruoder) werden wie die starken masculina flectiert; an diese $r$-stämme wahrscheiulich sich anlehnend auch die ursprünglichen $n$-stämme $f \circ{ }^{2} t^{i} r$ (fetiro), gəfadr (gifatero), ebenso auch $d 0^{2} d r$ (totoro); tsańdr (zuntaro) wird blos im sing. gebraucht.

Anm. 2. bi 2 osm (bësamo), dîsm (deismo), par ${ }^{2} d m$ (pfëdemo) sind aus der $n$-declination in die starke tubergegangen; ebenso auch $s t^{\dagger} \alpha^{2} r n$ (stërno).

Anm. 3. $f o^{2} g^{2} n t^{\prime}$ ( fiant) und froent' (friunt) flectieren stark; der plural ist gleich dem singular.

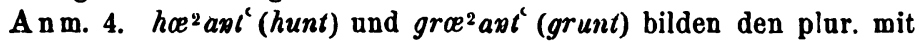

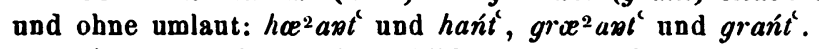

A n m. 5. dyurn (dorn) bildet den plur. de $e^{2} r n$ und $d e^{2} r n r$. - $h i^{2} r t^{6}$ und $r i^{2} s$ (hirti, risi) flectieren in plur. schwach: $h i^{2} r\left(n, r i^{2} z n\right.$; stark dagegen : $r k^{2} k^{2}$ (hrukki).

b) Schwaches masculinum.

$\S 110$. In den meisten fällen ist das bezeichnende $n$ auch im singular erbalten. Je nachdem, ob im plural umlautung stattindet, oder nicht, erhalten wir die beiden paradigmata:

\section{Sing.}

Plur.

N. G. D. A. k'yuln, branən $k^{c}$ yuln, bræ2nən.

Anm. 1. In vielen fällen schwankt der plural zwischen der umgelauteten und der nichtumgelanteten form; so in byuzn (bogo), byurin (borto), brôdn (bralo), do ${ }^{2} m \partial n$ (dûmo). Stets obne umlaut ist der plur. von bo ${ }^{2}$ olk'n (balko), bo²dn (boto), gald'jn (galgo), hùfn (hûfo, houf), $k^{\prime} y u l n$ (cholo), $k^{\prime} a^{2} a x n$ (chuohho). Immer umgelautet erscheint er in branan - brac nan (brunno), fladn - flac ${ }^{2} d n$ (flado), gu'artn - gậrtn

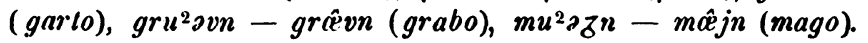

An m. 2. Die unlantsunfähigen schwachen masculina zeigen in der 
mundart natürlich keine flexion; sing. und plur. $z i^{2} \ln (s i l o), \grave{s} l i^{2} d n(s l i l o)$, rêman (riumo) u. s. w.

$\S 111$. Fur diejenigen schwachen masculina, welche nur im plural das ursprüglich stammhafte $n$ zeigen, ergibt sich folgendes paradigma.

Sing.

Plur.

N. G. D. A. yus, bi² 2 r yusn, bi² 2 rn

Anm. 1. Zwischen formen mit und ohne $n$ im singular schwanken $h u^{2} \partial s$ (haso) und $h u^{2} n$ (hano) - hutozn, $h u^{2} n a n$. - Stets obne $u$ im

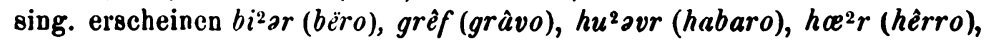
müaxi (mâgo), môn (mâno), nu'avl (nabolo), yus (ohso), re ê (rîfo), zòm (samo), faf (pfaffo). - kiyul (cholo), vgl. \$110, flectiert nach \$114.

Anm. ?. arm (arm) und halm (halm) sind aus der starken in die schwache declination übergegangen und bilden den singular auch wit $n$ : armon, halmon, plur. armon, halmon.

\section{Die feminina.}

$\S$ 112. Die historischen unterschiede der declinationsweisen des femininums sind in der mundart derart verwischt, dass nur noch eine grenze gezogen werden kann zwischen der alten $i$ - [und $u$-] klasse einerseits - diese allein flectiert noch stark -- und der alten $a$ - und $n$-declination anderseits, welche letzteren sich so durchdrungen haben, dass wie im nhd. der singular stark, der plural schwach flectiert.

a) Starkes femininum.

$\S 113$. Sing.

Plur.

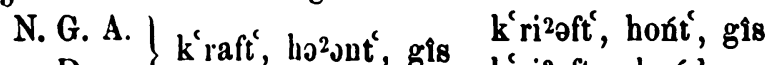
D. K k raft, hoºnt, gîs k'ri2oftn, hońdn, gîsn

Anm. 1. Folgende, meist umlautsunfähige feminina bilden den plur. auf $n$, obwol sio \%ur $i$-klasse gehøren: arbat (arabeit), bre $e^{2} \mid f t^{c}$

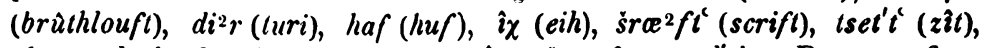
plur. arbadn, bre ${ }^{2} l f^{\prime}\left(n, d i^{2} r n\right.$, hafn, ìn, šrce ${ }^{2} f t n$, tsed'dn. Dagegen flectieren durchweg stark: brast (brust), flyu (flôh), foest (füst), go ${ }^{2} \mathrm{o}^{2} \mathrm{~s}$

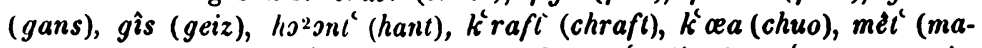

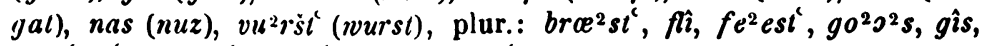

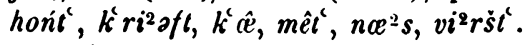

An $\mathrm{m}$. 2. Die verwantschaftsnamen $m o^{2} t^{2} r$ (muoter) und dùxír (tohter) werden stark flectiert; $z a^{2} s i^{i} r$ (swëster) dagegen bildet den plur.

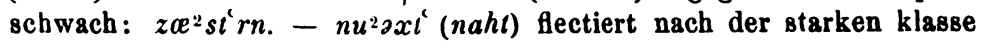
pl. nốxt ${ }^{6}$.

A n m. 3. Der plural von $b r a^{2} o^{2} k^{2} t^{c}(b r u \hat{u} t)$ und $h a^{2} o^{2} k^{2} t^{c}(h u \hat{u} t)$ wird 
$b r a^{2} o^{2} k^{\prime}$ ln, $h c^{2} o^{2} k^{2}$ in gebildet. Wenn man hierzu $\$ 71,3$ vergleicht, wozu ich noch anführe, dass es mir immer schwierigkeiten bereitet, den plural von diesen beiden wörtern zu bilden, und ich ihn häufig $b r e^{2} k^{i} t$, $h e^{2} k^{2} t^{t}$ gebildet habe, so lässt sich leicht erkennen, wie die gramnatischen kategorien in der lebenden sprache in steter berwegung sind, so dass sich solche scharf überhaupt nicht von einander scheiden lassen.

b) Gemischte feminin-declination ( $a$ - und $n$-klasse).

\begin{tabular}{|c|c|c|}
\hline 114. & Sing. & \\
\hline N. G. D. A. & k'vuªl (quâla) & $k^{\prime} v^{2} 2 \ln$ \\
\hline$"$ & zíl (sêla) & zîln \\
\hline$"$ & î (êwa) & îon \\
\hline$"$ & $k^{c} e^{2} n \partial x g x^{2} n$ (kuningîn) & $k^{\prime} e^{2}$ nexgat2nən \\
\hline$"$ & zańt (sunta) & zańdn \\
\hline " & tsæ2a ${ }^{2} a x$ (zunga) & tsæ2a $a p \partial n$ \\
\hline$"$ & frồ (frouwa) & frê̋ən \\
\hline " & hî (hôhî́) und he $e^{2} \chi t^{\dagger}$ & - \\
\hline & brît' (breitî) & \\
\hline
\end{tabular}

A $\mathbf{n}$. 1. Von alten nominalabstractis auf $-\hat{i}$, welche von den alten verbalabstractis auf -in beeinflusst wurden, kenne ich in der mundart nur $k^{\prime} a^{2}$ aldn (cheltî und cheltin).

A n m. 2. Nach der gemischten declination flectiert $z e^{2} s i^{2} r$ (swëster), vgl. $\$ 113$ a. 2 , und $b u^{2} r \chi$ (burg) : zact st $r n, b u^{2} r j n$. Wahrscheinlich ans dem grunde, weil sie meist im plural gebraucht werden, sind in diese declination übergetreten die ursprïnglich schwachen masculina flôk (floccho), graef (griubo), vîs (weiso); aus demselben grunde auch die tiernamen mat' (mado), šnôk (snekko), šnĉp (snepfo), pó (pfâwo)? Die ahd. starken masculina hag, loc sind im dialekt feminina geworden: $h u^{2} \partial x, l \hat{o} k^{2}-h u^{2} \partial z^{n}, l \delta k^{2} n$.

\section{Die neutra.}

\$ 115. Dic neutra zerfallen in der mundart in zwei klassen: zu der einen gehören alle historischen starken neutra; diese bilden den plural consonantisch auf $-r$; zu der andern gehören die bekannten wenigen schwachen neutra des ahd., von denen wanga jedoch im dialekt fehlt.

a) Gemischte declination der neutra (histor. starken neutra).

$$
\begin{aligned}
& \S 116 \text {. Sing. }
\end{aligned}
$$

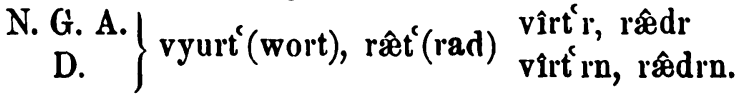


A n in. 1. Einige neutra bilden den plural nicht auf $-r$, sundern flectieren auch in diesem numerus stark; es sind teils solche, welche meist im plural gebraucht werden, teils solche, deren singular bereits eine pluralische bedeutung gewinnen kann.

$$
\begin{array}{ll}
\left.\begin{array}{c}
\text { Sing. } \\
\text { N. G. A. } \\
\text { D. }
\end{array}\right\} \begin{array}{l}
\text { Plur. } \\
\text { ru }^{2} \partial s, \text { hôr }
\end{array} & \begin{array}{l}
\mathrm{ru}^{2} \partial s, \text { hôr } \\
\mathrm{ru}^{2} \partial s n, \text { hôrn. }
\end{array}
\end{array}
$$

Hierher gehören: rüas (ros), šôf (scâf), šveń (snîn), dìl (teil), - hôr (hâr), k'yurn (chorn), bryut' (brôt), śpyur (spor), vasr (wazzar). Die beiden letzten werden überhaupt nur im sing. gebraucht. Jedoch hürt man auch $r u^{2} \partial s-r i^{2} \partial s r$, dîl - de $e^{2} l r$, bryut $-b r \hat{\imath} d r$, besonders in der bedeutung 'nicht zusammengehörige pferde, teile, brote'.

Anm. 2. Auf $-r$ bilden alle deminutiva den plural: met' $\chi r$ (mädchen), $l e^{2} f k^{\prime} r$ (liebchen), rańlt' $\chi r$ (ringlein).

An m. 3. Das ursprüngliche masculinum mènat (manôd) erscheint im dialekt als neutrum, bildet den plural aber ohne $r$.

b) Schwaches neutrum.

§ 117. Sing. N.G. D.A. ûx (auga), yur (ôra), hææ2 ${ }^{2}$ rts (hërza) Plur. " âzn, yurn, hæ2 rtsn.

A nm. Neben hacrts hürt man auch haertsn; in den beiden andern wörtern erscheint das $n$ nie im singular.

\section{Anomala.}

$\S 118$. Von den sogenannten anomala hat nur man eine sonderstellung behaupten können (vgl. § 109 a. 1 u. 3 , §113 a. 2, § 114 a. 2), es flectiert:
N. G. A.
D. $\}$ mo2on
Plur.
mo2on
mo23non.

\section{Flexion der eigennamen.}

§ 119. Eigennamen werden einzelstehend nie flectiert; jedoch hat sich die spur einer flexion erhalten bei der zusammenstellung von zu- und vornamen. Es wird nämlich im dialekt der familienname stets vorangesetzt: Müller Johann. Ist nun derselbe einsilbig, so bekommt or die schwache (genetiv-) endung $n$, welche vor andern lauten als $d, t, h$ und vocal (vgl. § 9) schwindet und ein a zurücklässt. Ist der familienname jedoch mehrsilbig, so bleibt er flexionslos, es sei denn, dass er auf der ultima betont sei. - Diese rein 
mechanischen verhältnisse - niemand fühlt hier eine flexion - lassen gerade darum keine ausnahme zu. Beispiele: Bieltz, Rolh, Hietsch, Wolff, Paur; Schuller, Wegendt, Schaffend; Andrä, Bardon (die beiden letzten oxytoniert): bîltsə Misch (Michael), rôdn Hans, hîtšn Dani (Daniel), vo²lvn Andres, p020ra Lotti (Lolle); dagegen šullr Hans, ve jnit Louise, šafnt Andres; aber widerum andrêan Titz (Andreas), bardo ${ }^{2}$ n Misch. Ebenso o ${ }^{2}$ rjo$n i^{2} \sin$ Dani (organisten Daniel).

Anm. Von einer flexion der städte- und ländernamen ist in der mundart keine spur vorhanden.

\section{B. Flexion der adjectiva.}

§ 120. Bei den adjectivis ist im dialekt nicht nur der unterschied der verschiedenen stämme völlig geschwunden, sondern auch die starke und schwache flexion der adjectiva hat sich einer ausgleichung unterziehen mussen. Neben dieser ausgeglichenen adjectivischen flexion besteht im gebrauch noch die 'unflectierte' form, welche in prädicativer stellung angewendet wird; die flectierte form des nominativs sing. ahd. - $\hat{e} r,-i u,-a z$, im dialekt $-r,-,-(\partial) i$, wird substantivisch nach dem unbestimmten artikel gebraucht. In substantivischem gebrauch nach dem bestimmten artikel flectiert der ganze plural schwach auf $-n$.

\begin{tabular}{|c|c|c|c|}
\hline $\begin{array}{r}\S 121 . \\
\text { S. N. }\end{array}$ & $\begin{array}{l}\operatorname{masc} . \\
\text { bîis, -r }\end{array}$ & & $\begin{array}{l}\text { neutr: } \\
\text { hîšs, -ət' }\end{array}$ \\
\hline G. & & hîšn & \\
\hline D. & & bišn & \\
\hline A. & bǐ̌n & & bis, -et \\
\hline PI. N. & & & hî̉ \\
\hline G. & & & hนิรัน \\
\hline D. & & & hîsn \\
\hline A. & & & hǐš \\
\hline
\end{tabular}

Anm. 1. Wie hiš (mhd. hövesch) flectieren alle adjectiva. Der flectierte nom. sing.: o-blaridr (ein blinder), on-blarit (eine blinde), e-blańdal (ein blindes). Nach dem bestimmten artikel lautet in substantivischem gebrauch der ganze plural hîšn.

Anm. 2. Die comparation. Der comparativ wird gebildet durch anhängen der endung -(a)r, der superlativ durch die endung -st, wobei ausser $\S 93,4$ und $\S 104,5$ noch zu berïcksichtigen ist, dass auslautendem $\check{s}$ das $s$ in $s$ sich assimiliert. Umlaut tritt nach anhängung 
dieser endungen nicht immer ein; ich bin jedoch ausser stande, hierfür eine bestimmte regel anzugeben. Offenbar muss unterschieden werden zwischen den fällen, wo comparativ und superlativ stets oder von den meisten neugebildet, nnd denjenigen, wo ihre formen überkommen werden. Bei den ersteren glaube ich ein positives sträuben gegen den umlaut constatieren zu dïrfen.

Als beispiele für das oben bezuiglich der superlativendung -st angeführte, mögen dienen: $i^{2} r r^{\prime} t^{t}$ (erste), vorršt (wahrste), $t^{t} u^{2} m t s t^{\prime}$ (dümmste), $l e^{2} z t s t^{i}$ (lïngste), $h e^{2} \check{s}^{i} t^{\prime}$ (hübscheste).

Ohne positiv sind $b i^{2} a s r, b i^{2} a s t^{\prime}$ (bezziro, bezzisto), mî, mîst' (mèro, meisto), ohne positiv und comparativ li2atst' (lezzisto).

Anm. 3. Das adverb ist in der mundart lautlich gleich der unflectierten form des adjectivums. Verschieden von diesem ist es in $l 0^{2} n$ adv. zu $l 0^{2} x k^{\prime}$ (lang) vgl. $§ 106,2$; šyun (scôno) wird nicht mehr mit šin (scôni) in zusammenhang gebracht, vgl. $§ 77,2$, ebensowenig fast mit $f^{2}$ ast (festi), vgl. $\S 34,4$. Vom adverbium ist die unflectierte form des adjectivums beeinflusst in hart ${ }^{2}$ (herti) und gošvañt ${ }^{2}{ }^{*}$ swindo) vgl. \$ 45, 2. Comparativ und superlativ des adverbs sind gleich den unflectierten formen der adjectivischen steigerungen.

\section{Flexion der pronomina und zahlwörter.}

\section{Declination der pronomina.}

§122. Unter dem einfluss des satzaccents haben sich zu den meisten formen der pronomina nebenformen gebildet, welche nur in unbetonter stellung im satze angewendet werden, während die volleren bauptformen gebraucht werden, wenn der nachdruck auf dem pronomen ruhen soll. Im folgenden werden beide stets durch einen strich getrennt erscheinen, da wo jene differenzierung uberhaupt stattgefunden hat.

a) Ungeschlechtiges pronomen.

\section{1. pers.}

Sing. N. $e^{2} \chi-i^{2} x, x$

G. [meńr]

D. $\mathrm{mi}^{2} \mathrm{r}-\mathrm{mər}, \mathrm{mIr}$

A. $\mathrm{me}^{2} \chi-\mathrm{mi}^{2} \chi$

Plur. N. mi ${ }^{2} r-m ə r, m r$

G. [æazr]

D. A. xas - əs
2. pers.

Reflexivum

$$
\begin{aligned}
& \text { [deńr] } \\
& \mathrm{di}^{2} \mathbf{r}^{\mathrm{r}}-\mathrm{d} \boldsymbol{\mathrm { d }}, \mathrm{dr} \\
& \operatorname{de}^{2} \chi-\operatorname{di}^{2} \chi \\
& \mathrm{i}^{2} \mathrm{r}-\mathrm{er}, \mathrm{r} \text {, } \\
& \text { [i²rir] } \\
& \mathrm{e}^{2} \chi-\mathrm{i}^{2} \chi, \chi \\
& \begin{array}{c}
- \\
\mathrm{ze}^{2} \chi-\mathrm{zi}^{2} \chi \\
\mathrm{ze}^{2} \chi-\mathrm{zi}^{2} \chi
\end{array}
\end{aligned}
$$

$A \mathrm{n}$. Die eingeklanmerten genetive sind nicht volkstümlich und werden nur von solchen gebraucht, welche viel hd. sprechen. 
b) Geschlechtiges pronomen.

§ 124. 1. Pronomen der dritten person. masc. neutr.

fem.

Sing. N. $h^{2} \partial-\theta \quad x^{2} t^{c}-\theta t^{t} \quad z \hat{x}-z \theta$

G. [zeńr] [zeńr $]$ [æ2rər]

D. $\quad \mathfrak{x}^{2} \mathrm{~m}-$ əm, m $\quad \mathfrak{x}^{2 \mathbf{r}}-$ ər, r

A. $x^{2} n-\partial n, n \quad æ^{2} t^{t}-\partial t^{t} \quad z \hat{\mathscr{E}}-z \partial$

Plur. N.

$\mathbf{z} \hat{\mathscr{X}}-\mathbf{z o}$

G.

$\mathfrak{x}^{2} \boldsymbol{r}^{*}$

D.

$æ^{2}$ nən - ən

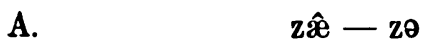

$\S$ 125. 2. Einfaches demonstrativpronomen. masc. neutr.

fem.

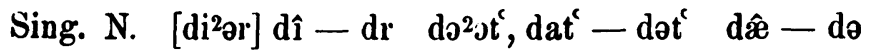
G.
$d^{2} \partial s-d \partial s$
$\mathrm{di}^{2} \partial \mathrm{r}-\mathrm{dr}$
D.
$\mathrm{di}^{2} \partial \mathrm{m}, \mathrm{de}^{2} \mathrm{~m}-\mathrm{d} \partial \mathrm{m}$
$\mathrm{di}^{2} \partial \mathrm{r}-\mathrm{dr}$
A. $d^{2} \partial n-d n$

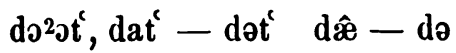

Plur. N.

G.

d $\hat{\ddot{X}}$ - do

D.

$d i^{2} \partial r-d r$

A.

$d^{2} \partial n-d n$

$\mathrm{d} \hat{\ddot{X}}$ - də

A n m. Die vollen formen werden hinweisend, die abgeschwächten als artikel verwendet. dat wird nie als demonstrativum, sondern wie hd. 'dass' gebraucht.

$\S 126$. 3. Zusammengesetztes demonstrativum. masc. neutr. fem. Sing. N. de ${ }^{2} r \quad d^{2} t \quad d e^{2} s$

$\begin{array}{rrrr}\text { G. } & d e^{2} z \theta s & \\ \text { D. } & d e^{2} z m & d e^{2} z r \\ \text { A. } & d e^{2} z n & d e^{2} t & d e^{2} z r \\ \text { Plur. } & \text { N. } & d e^{2} s & \\ \text { G. } & d e^{2} z r & \\ \text { D. } & d e^{2} z n & \\ \text { A. } & & d e^{2} s . & \end{array}$

A $\mathrm{nm}$. Von diesen pronomen, wie auch vom folgenden, gibt es nur volle formen, da stets der satzton auf ihm ruht. 
$\S 127$. 4. Interrogativum.

$$
\begin{aligned}
& \text { masc. } \\
& \text { Sing. N. [vi² } \left.{ }^{2} r\right] \text { vî vo } 20 t^{t}, \text { vat } \\
& \text { G. } \quad \mathrm{i}^{2} \partial \mathrm{s} \\
& \text { D. } \quad i^{2} \partial m, v e^{2} m \\
& \text { A. vi² } \quad \text { vo }^{2} \partial t^{\mathrm{i}} \text {, vat }
\end{aligned}
$$

A n m. Die form $v v^{2} \jmath^{\prime}\left(\nabla \mathrm{gl} . \S 125 d^{2} \partial t\right)$ ist nicht so selr die ältere, als die vollere form; sie wird gebraucht, wenn die widerholung einer frage gewünscht wird: $v g^{2} t^{\prime}$ ? (was denn?); doch wird auch hier immer häufiger vat gebraucht. Für den genetiv vi'as (vi2azeń? wess' sein?) wird meist der gen. des pronominaladjectivums $v e^{2} l r$ (welcher) gebraucht, $v e^{2} l a s$ (ve 2 lezen? welches sein?).

§ 128. 5. Relativum. Fur das feblende relativum wird das einfache demonstrativum gebraucht. An den nominativ aller geschlechter und numeri wird jedoch - $d n$ (ahd. $d \hat{a} r, d e$ ) angehängt: $d \hat{\imath} d n, d 0^{2} v_{v} d n, d \hat{e} d n$; für $d 0^{2} v_{v} d n$ wird auch $v e^{2} l_{v} d n$ (welches) gebraucht und fưr dî̀dn auch vîdn (wer).

c) Pronominaladjectiva und indefinita.

$\S 129$. Die pronominaladjectiva und indefinita unterscheiden sich in der flexion von den adjectivis dadurch, dass sie den gen. dat. sing. aller drei geschlechter stark bilden, und zwar die possessiva und ein, jënêr, hwelhh, ioman, nioman nur stark, wälrend die andern in nicht-substantivischem gebrauch wio die adjectiva decliniert werden.

$\S 130$. Die possessiva lauten im dialekt: $m e n ́, m e^{2}$ (vgl. $\S 9)$, deń, zeń; aeas, $i^{2} r, a^{2} r$.

$$
\text { masc. neutr. fem. }
$$

Sing. N. meń, -ər, me meń, - $t^{2}, m^{2}$ meń

G. meñes meńr

D. meńom meńr

Plur. N. meń

A. meńən meń, -t', me meń

G. meńl

D. meńən

A. meń

A $\mathbf{n m}$. Der flectierte nominativ sing. wird nur substantivisch ohne artikel gebraucht: meńr, $i^{2} r a r$ der meinige, eurige.

$\S 131$. An weiteren pronominaladjectivis und indefinitis sind anzufuhren: 
1. ein: masc. $\hat{\imath} n,(\hat{\imath}), e^{2} n r$, neutr. $\hat{\imath} n,(\hat{\imath})$, int , fem. in.

A n m. Die abgeschwächten formen von ein : on, a bilden den unbestimmten artikel. Dieser flectiert:

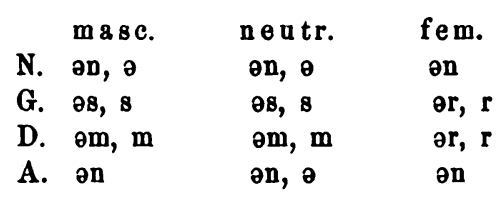

Ebenso flectiert ein auch in den folgenden zusammensetzungen, wenn dieselben in unbetonter satzstellung sich befinden.

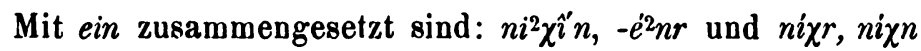
(nihein) und davon abgeleitet $i^{2} \chi r, i^{2} \chi n$ (irgend einer); $z \dot{o}^{2} l \hat{\imath} n$, $-e^{2} n r$ (sulîh - ein), daneben auch unter hd. einfluss a-zói $\chi \hat{\imath} n$, $-e^{2} n r ; \quad v \delta^{2} l \hat{\imath} n,-e^{2} n r(h w e l i \hat{h}-e i n) ; \hat{\imath}^{\prime} k^{\prime} l i^{2} j e ́ n r$ (iogilìh - einêr 'jeder ohne ausnahme'); di²arîn, $-e^{2} n r$ (dëro - ein 'einer von jenen'); $\hat{\imath}^{\prime} d r i^{2} j e^{2} n \partial s$ und $-e^{2} n r$, gen. sing. und plur. (iedër, -ig-, ein) 'allerhand'.

2. al: alant, alant r (allez); acc. sing. masc. alan (alon $d 0^{2} \supset x$, jeden tag), plur. alan, Fur alont wird unter dem einfluss des nhd. meist alos gebraucht.

3. Zu ahd. wiht: noe $e^{2} s t$ und $a^{2} s t$, auch $n e^{2} s t^{t} r$ und $a^{2} s t^{t} r$, 'nichts, etwas'.

4. ëtalîh, ëtteslih: $\hat{e} t l i^{2} \chi$, $\hat{e} t s l i^{2} \chi$, nur im plural gebräuchlich.

5. man. Als indefinitum lautet man im dialekt $\partial m, m$. Mit man zusammengesetzt $n e^{2}$ mast ${ }^{c},-r$ und $e^{2}$ mast, $-r$ (nioman und ioman); flectiert dat. ne'mestam und -stram, acc. -stan und -stran.

6. Zu abd. sëlb: indeclin. záe ${ }^{2}$ alvast ${ }^{t}$ und $z e^{2}$ alvasi $r$.

7. andar: $0^{2}$ ondr, wird nur adjectivisch flectiert. Starker gen. sing. nur im adverb $\rho^{2}$ sndrš 'anders'.

8. jënêr: masc. $g e^{2} n\left(g e^{2}\right), g e^{2} n r$; neutr. $g e^{2} n\left(g e^{2}\right), g e^{2} n t$; fem. $g e^{2} n$.

9. iedër: masc. jêt', jêdr; neutr. jêt', jêdat; fem. jêt . Meist mit dem unbestimmten artikel verbunden: $a-j \hat{e} t, a-j e \hat{t}$, an-jêt'.

10. hwelîh: masc. $v e^{2} l, v e^{2} l r ;$ neutr. $v e^{2} l, v e^{2} l l^{i} ;$ fem. $v e^{2} l$.

11. sulih : nur in der zusammensetzung s. oben unter 1.

12. èogilih : nur in der zusammensetzung vgl. oben unter 1 . 


\section{Die zahlwörter.}

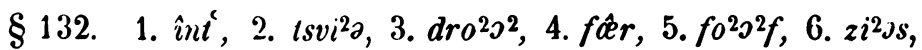
7. $z i^{2} v n$, 8. $\hat{a} \chi t^{t}, 9 . n e n ́, 10 . t s \hat{c} n, 11 . e^{2} l f, 12 . t s v e^{2} l f, 13 . d r e^{2-}$

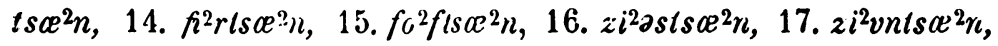
18. $u^{2} \partial x t s \alpha^{2} n$, 19. nénts $\alpha^{2} n$, 20. tsvi ${ }^{2} n t s i^{2} \chi, 21$. înantsvi ${ }^{2} n t s i^{2} \chi$, 22. tsvinantsvi ${ }^{2}{ }_{u} t s i^{2} \chi$ u. s. w., 30. tre $^{2} e s i^{2} \chi, 40 . f^{2} r t s i^{2} \chi, 80 . u^{2} e x-$ tsi $i^{2} \chi, 100 . h e^{2} a x d r i, 1000$. i $\ltimes o z n i$.

Ordinalia: 1. $i^{2} r s t^{t}$, 2. $t s v i^{2} \partial t^{\mathrm{c}}, 3 . d r e^{2} t^{\mathrm{t}}$, 4. $v i^{2} r t^{\mathrm{c}}$ u. s. w.

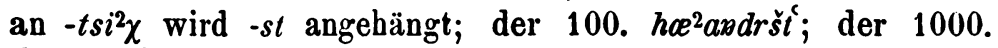
ícozntst.

$\S 133$. Die ordinalia werden wie die adjectiva flectiert, die cardinalia ausser 1. und 2. dagegen nur im dat decliniert durch die dativendung $-n$, bei substantivischem gebrauch.

Die flexion von ein ist $\S 130$ angegeben. Die zweizahl lautet im masc. tsvin, neutr. $t s v i^{2} \partial$, fem. $t s v \hat{o}$, der dat. $t s v \varkappa^{2} n a n$, wenn die zahl substantivisch, tsvoe"n, wenn sie adjectivisch gebraucht wird.

\section{Flexion der verba.}

$\S$ 134. Die beiden klassen der starken und schwachen verba sind formell noch sehr scharf getrennt; von den unterabteilungen der schwachen conjugation bebt sich ein teil der -ja-klasse dadurch ab, dass der 'ruckumlaut' in ihn noch volle geltung hat.

Von den modis des ahd. verbums ist der conjunctiv praesentis lautlich durch den indicativ desselben tempus, syntactisch besonders durch den conjunctiv praeteriti verdrăngt worden. Dagegen ist noch das gerundium erhalten, freilich nur im genetiv und auch hier nur in wenigen redensarten.

A nm. Bezilglich des 'grammatischen wechsels' vgl. \$137 a) b), $\S 140$ a), $\S 141 \mathrm{e}), \S 142$ b) c).

I. Tempusbildung der starken verba.

$\S 135$. Für den wichtigsten hier in betracht zu ziehenden punkt, den ablaut, sind ganz wol noch die ablautsreihen des abd. massgebend. Die ablautsstufen des sing. und plur. praet. sind ubcrall ausreglichen; dagegen hat sich in gewissen fällen der unterschied zwischen gebrochenem und ungebrochenem praescnsrocal erhalten. Es nulissen demnach im folgenden an- 
gegeben werden: 1. Der infinitiv, flir das ganze praesens (mit ausnahme des imperativs im singular und der II. III. pers. sing. ind. 2. Der imperativ). (3.) 2. Dic I. pers. sing. ind. praet. (4.) 3. I. pers. sing. conj. praet. (5.) 4. Das participium praeteriti.

$\S 136$. I. Ablautsreibe, abd. $\hat{\imath}-a i-i-i$. Diese reibe hat sich im dialekt gespalten in folgende unterabteilungen:

a) $e^{2} e-i^{2}-i^{2}-i^{2}$, vgl. $\S 44,2$ und $\S 65: b l e^{2} e v n-$ $b l i^{2} f-b l i^{2} f-g a b l i^{2} v n$. So gehen: ble evn (biliban), dre $e^{2} e v n$ (trîban), bə-k'le $e^{2} e u n$ (chliban), re ${ }^{2} e u n$ (rîban), šr $e^{2} e u n$ (scrîban).

b) $e^{2} e-\hat{\imath}-\hat{\imath}-a^{2}$, vgl. $\$ \S 42.65 .75: b e^{2} e s n-b \hat{\imath} s-$

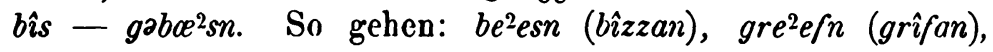
$r e^{2} e s n$ (rîzzan), šme $e^{2} e s n$ (smîzzan), še $e^{2} e s n$ (scîzzan), $1 s a-s ̌ l o e^{2} s n$, part. praet. von ahd. shizzan.

c) $e-i^{2}-i^{2}-i^{2}$, vgl. § 63: šned dn- šni $i^{2} \iota^{\prime}-\check{s} n i^{2} t^{t}$ - gešni $i^{2} d n$, šeñan - ši $i^{2} n-\operatorname{si}^{2} n-g \partial \check{s}^{2} n \partial n$. So gehen: red'dn (rîtan), šned'dn (snîdan), šred'dn (scrîtan), šlred'dn (strîtan); greñan (grînan), šeñan (scînuri).

An m. 1. $k^{2} 2^{2} n a n$ (kinan) ist sicher von dem part. priet. ant-kik $i^{2} n a n$ beeinflusst, welches hauptsächlich gebraucht wird.

d) $e^{2}-i^{2}-i^{2}-i^{2}$, vgl. $\$ 64:$ š $e^{2} j n-\check{s}^{t} i^{2} \chi-\grave{s}^{2} i^{2} \chi$ - gošt $i^{2} j n$. So gehen: št $e^{2} j n$ (stîgan), ve $e^{2} \chi$ (nîhhan) und das ursprunglich schwache šve'jn (swrigên).

An $n$. 2. $\dot{s}^{2} c^{2} \chi^{n}$ (slihhan) ist in die II. ablautsreihe ubergegangen. Auch zu sit $e^{2} j n$ wird das praet. zuweilen $\check{s}^{2} u^{2} x$ gebildet.

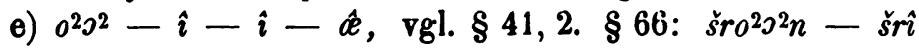
- šrî - gašr (ên. So gehen: $d 0^{2} 0^{2} n$ (dîhan), $l^{2} J^{2} n$ (lîhan), špo $0^{2} \rho^{2} n$ (spiwan), šro $0^{2}{ }^{2} n$ (scrian). So auch $r^{2} \jmath^{2} n$ (riuwan).

\$ 137. II. Ablautsreibe, ahd. io, iu - au - u-o. Diese reibe spaltet sich in der mundart in folgende:

a) $a-e^{2} e-u^{2}-i^{2}-y u$, vgl. $\$ \S 51.82 .82$ a. 2 : frôzzn - fre $e^{2} e s !-f r u{ }^{2} r-f r i^{2} r-g a f r y u r n$. So gehen: frêzzn (friosan), forlêezn (farliosan).

A nm. 1. nâzzn (niosan) wird schwach conjugiert; auch der imper. lautet $n \hat{e ̂ s}$ ! Ebenfalls schwach geworden ist št $\hat{x} \hat{e} v n$ (stiuban).

b) $a^{2}-e^{2}-u^{2}-i^{2}-y u$, vgl. $\$ \S 51.80 .81: l a^{2} j n$ - $l e^{2} \chi !-l u^{2} x-l i^{2} \chi-g a l y u z n$. Hierber gehören: badra ${ }^{2} j n$

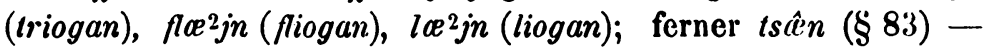

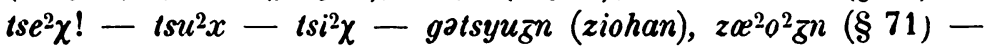
$z a^{2} o^{2} x !-z u^{2} x-z i^{2} \chi-g \partial z y u \bar{z} n$ (sügan). 
c) $a^{2}-e^{2}-u^{2}-i^{2}-\hat{o}$, vgl. $\S \S 48.80 .81: r a^{2} \chi^{n}-$

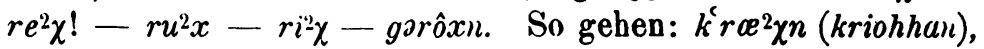
$r e^{2} \chi n$ (riohhan), šl le $\chi \chi$ (slihhan, vgl. $§ 136$ a. 2), dazu $z 0^{2} f n$ (§ 72 a. 1) $-z o^{2} f !-z u^{2} f-z i^{2} f-g \ni z \hat{o f n}$ (sûfan).

d) $e^{2}-e^{2}-u^{2}-i^{2}-u \partial$, vgl. $\$ \S$ 49. 80: $g e^{2} s n-$ $g e^{2} s !-g u^{2} s-g i^{2} s-g a g u^{2} \partial s n$. So gehen: far-dre $e^{2} s n$ (driozzan), ge $e^{2} s n$ (giozzan), fle ${ }^{2} s n$ (fliozzan), ga-ne ${ }^{2} s n$ (niozzan), šle ${ }^{2} s n$ (sliozzan).

A nm. 2. $\check{s}\left(e^{2} s n\right.$ (slizzan) in der bedentung 'federn entkielen' ist in diese klasse ibergegangen; in der bedeutung 'zerschlitzt' wird von einem zu erwartenden šle'esn das partic. $t s o s ̌ l l e^{2} s n$ gebraucht (vgl. $\S 136 \mathrm{~b})$.

e) $e^{2}-e^{2}-u^{2}-i^{2}-o^{2}$, vgl. $\$ \$$ 52. 79: hed dn (biotan) - bet $t^{\prime} t$ - $b u^{2} t-b i^{2} t^{t}-g a b o^{2} d n$.

A $\mathrm{nm}$. 3. $r 0^{2} g^{2} n$ (riuwan) ist in die I. ablautsreihe tibergegangen (vgl. $\$ 136 \mathrm{e}$ ); $k^{2} o^{2} g^{2} n$ (chiuwan) flectiert schwach; das partic. wird anch stark gebildet gok' ân.

$\S 138$. 1II. ablautsreihe $\ddot{e}, i-a-u-u$, o. Diese reihe zerfällt in der mundart in folgende abteilungen:

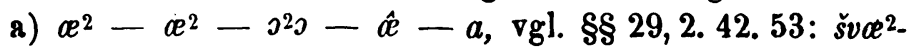

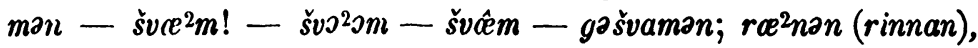

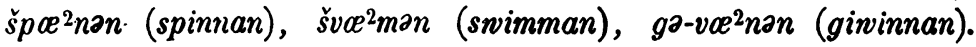
Ebenso auch gaenan (gi-unnan) und ša $e^{2}$ nan (scintun) vgl. § 104,6.

Anm. 1. Ahd. linnan fehlt im dialekt; erhalten ist dagegen das zugebörige causativum $l e^{2} n \partial n$, praet. $l 0^{2}$ ont $t^{2}$ eine zumutung, eine beschuldigang von sich abweisen'. rae'non (rinnan) wird nur vou leblosen dingen gebraucht; von lebenden wesen $r e^{2} n o n, r o^{2} o n t^{4}$ (rennen). brân, ein schw. zeitwort, vercinigt die bedeutung von brinnan und brennen in sich. - do $0^{2} g^{2} z n$ (§ 47 ) ahd. dinsan flectiert entweder schwach oder nach $\$ 136$ e).

b) $a-a-o^{2}-a^{2}-a^{2} a$, vgl. \$\$ 31. 45. 57: zañan-

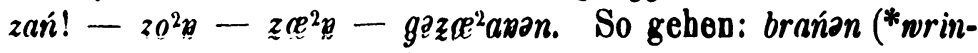
gan), drañan (dringan), k'lañan (chlingan), ga-lańan (gilingan), zańan (singan), tsvañan (dwingan), ebenso auch die ursprlinglich schwachen an-tsańan (zunten) 'anziinden', trans. und intrans., vgl. §104,6; dañan (mldd. dingen).

$\mathrm{A}$ nm. 2. Für drańan und tsvañan werden im prïsens häufig die za ihnen gehürigen schwachen drorian und tsvonian gebraucht.

c) $a-a-0^{2} \supset-\hat{a}-a^{2} a$, vgl. $\$ \S 29,3.45 .57$ : $a \dot{a} d n$ - fanit! - fo2ont - fầnt - facasdn, bandn (bintan), fańdn (findan), far-švanidu (swintun). 
Anm. 3. fańdn und $k^{2} u^{2} n$ (quëman) sind die einzigen verba, welche das participium praeteriti ohne ge- bilden: faeandn, $k^{k} u^{2} n$.

d) $a^{2}-a^{2}-o^{2}-a^{2}-a^{2} a$, vgl. \$§31. 42.5i: $d r a^{2} b k n$

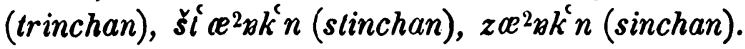

e) $a^{2} a-a^{2} a-\jmath^{2} \jmath-\hat{e}-a$, vgl. $\$ \S \div 9.36: h e^{2} a l f n$

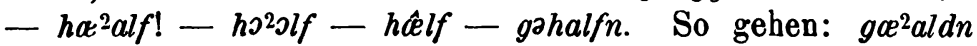
(gëltan), hœe alfn (hëlfan), šmcéaltsn (smëlzan), gašvœ2aln (swëllan). Ebenso auch dss ursprunglich schwache máaldn (mëldôn).

Anm. 4. bafiªln (bifelhan) ist in die 4. ablautsreihe übergegangen. bi2ln (bellan), vgl. $\$ 40 \mathrm{a.2}$, ist in die schwache conjugation übergetreten.

f) $a^{2}-e^{2}-u^{2}-i^{2}-o^{2}$, vgl. $\$ \S 34,2.44,1.52 .54$ : št $\alpha^{2} r v n-\check{s} e^{2} e^{2} r f !-s_{s} u^{2} r f-\check{s} t^{2} i^{2} r f-g \partial s^{2} \hat{o}^{2} r v n$. So gehen:

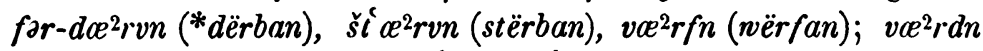

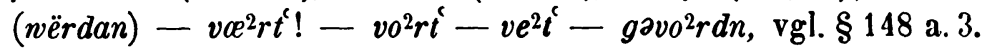

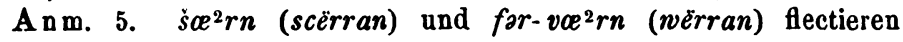
schwach oder sind mit den zugehörigen schwachen verben zusammengefallen.

$\S$ 139. IV. ablautsreibe ahd. $\ddot{e}, i-a-\hat{a}-0 . \mathrm{Zu}$ dieser reibe gebören folgende unterabteilungen:

a) $i^{2} \partial-i^{2}-u^{2}-i^{2 \partial}-y u$, vgl. $\S \S 40.44,2.51$ : št $i^{2} \partial \ln$ - št $i^{2} l !$ - ši $u^{2} l-\check{s}^{2} i^{2} \partial l$ - gašt yuln. So gehen: ši $i^{2}$ aln (stëlan); ferner bafíaln (bifëlhan), vgl. §138 a. 4, und švi2arn (sweren), vgl. §141 a.

b) $\hat{e}-a^{2}-\partial^{2} \partial-\hat{e}-\hat{o}$, vgl. \$§ 29. 38. 42. 48: brêen

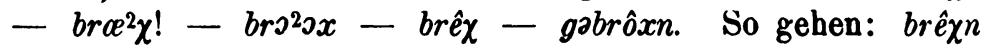

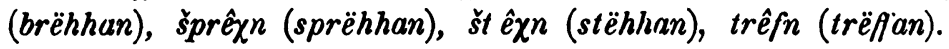

c) $n i^{2} \partial n-n e^{2} m !-n 0^{2} \mathrm{~s} m$ - nêm - ganyun (nëman),

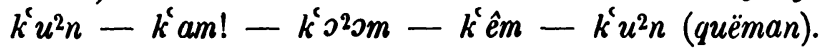

A n m. 1. driºšn (drëskan) und li2əšn (léskan) sind in die 5. ablautsreihe Ubergegangen.

A n m. 2. flí2 $\chi$ ln (flëhtan) und švi2 rn (swëran) flectieren schwach;

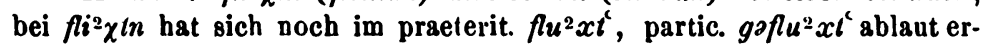
halten.

$\S 140$. V. ablautsreibe, abd. $\ddot{e}, i-a-\hat{a}-\ddot{e}$. Diese reihe zerfällt in folgende unterabteilungen:

a) $i^{2} \partial-i^{2}-\partial^{2} \rho-\hat{e}, \hat{e}-i^{2} \partial, \mathrm{vgl} . \S \S 40.44,2: \operatorname{tr} i^{2} \partial d n$ - $t r^{2} t^{2}--t r \partial^{2} \partial t-t r \hat{e} t^{c}-g a t r i^{2} \partial d n, l i^{2} \partial z n-l i^{2} s-l \partial^{2} J s$ - lês - gali2azn. Hierher gebören: ḱniªdn (clinëtan), triªdn (trëlan), $b i^{2} d n$ (bitten) - $b \partial^{2} \jmath t-b \hat{e} \iota^{i}-g a b \epsilon^{2} d n ; l i^{2} \partial z n$ 
(lësan); $10^{2} \jmath^{2} n($ ligen $)-l 0^{2} \jmath^{2} !-l 0^{2} J x-l e \hat{\chi}-g a l i^{2} a j n ; g i^{2} a n$

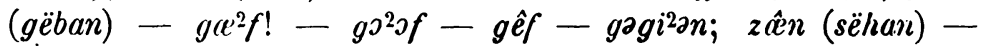
$z \dot{c}^{2} \chi !-z \partial^{2} 0 x-z \hat{e} \chi-g \partial z \hat{e} n$, ebenso gašên (giscëhan), vgl. $\S 41$ a. 2 .

A $\mathbf{n} \mathbf{m}$. Ueber ahd. wësan siehe $\$ 149$, d).

b) $i^{2} \partial-a^{2}-\partial^{2} \partial-\hat{e}-i^{2} \partial$, vgl. $\S 42: m i^{2} \partial s n-m a^{2} s$ !

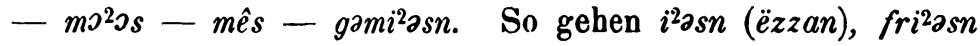
(frëzzan), far-gi²asn (firgëzzan), mi²asn (mëzzan); ebenso auch $d r i^{2} \partial$ šn und $l i^{2} \partial{ }^{2} n$, vgl. $\S 139$ a. 1.

$\S$ 141. VI. ablautsreibe, ahd. $a-\hat{o}-\hat{o}-a . \mathrm{Zu}$ berlicksichtigen sind $\S \S 30.33$. 34. 35, 1.67-69.

a) $m u^{2} a l n$ (malan), mi2allst - mu2al! - moeal - môel gamu2aln; so gehen fú2arn (faran), grü2avn (graban), šu2avn (scaban), mit umlaut im praesens hi2avn (heffan).

b) $v u^{2} \partial s n$ (wahsan), vi ${ }^{2} a s t-v u^{2} \partial s !-v 0^{2} s-v e^{2} s-g a-$ $v u^{2} \partial s n$; mit umlaut im praesens $v i^{2} \partial s^{2} n$ (wascan).

c) ladn (hladan) - lat! - laeat - lêt $t^{t}-g e l a d n$.

d) bakn (bacchan) - bak! - boe $a k^{k}-b a^{2} k^{k}-g \partial b a k n$.

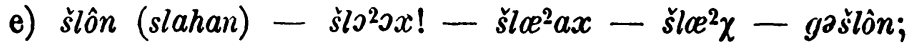
so noch drôn (tragan).

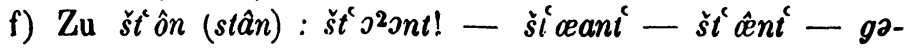
šloºndn.

A n m. šviarn (sweren) ist in die 4. reihe übergetreten, vgl. §139 a). - kiku'ozn (gnagan, nagan) flecticrt schwach. - spanan fehlt; erhalten ist nur das schwache $u^{2} \partial$ f-špênan 'den sängling entwöhnen'.

$\S 142$. VII. Reduplicierende verba.

a) hîsn (heizzan) - he's-gahîsn; šî̀dn (sceidan) - šceat, šêei - gašìdn.

A n m. 1. Wie hîsn gelit auch hîšn (eiscôn).

b) lafn (hloufan), lifst - le $e^{2} f-g$ lafn, št yusn (slôzzan),

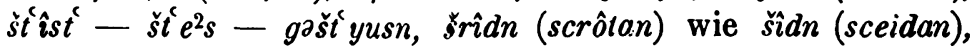
hôen (houwan) - hoe $a x$, hae ${ }^{2} \chi-g a h \hat{e} n$.

Anm. 2. šrîdn, vgl. $\S 77,2$, hat sehr specialisierte bedeutungen: die säge šrîdn 'die zähne der säge spitz feilen und auscinanderbiegen'; den weg šrîdn 'so fahren, dass die schlechten wegstellen zwischen die wagenräder kommen'. - Das in allgemeiner bedeutung gebrauchte šr $\partial d n$, praet. šrceat (wie von šrî̀dn) scheint sich an rôdn (râtan), vgl. unten d), angelehnt $\mathrm{zu}$ haben.

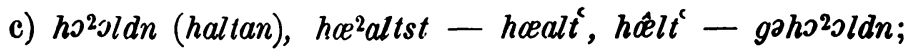

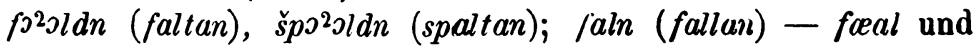


$f^{2} \mathrm{ol}-g a f a l n ; f e ̂ n(f a h a n)-f e^{2} x-g \partial f o^{2} x a n ;$ ebenso hên (hâhan) und zu gôn (gân) $g e^{2} x$ u. \&. w.

$\mathrm{A} \mathrm{nm}$. 3. šponan (spannan) ist schwach geworden.

d) šlôfan (slâfan) - šle $e^{2} f-g a s ̌ l o ̂ f n, ~ l o^{2} s n(l a z z a n)-l e^{2} s$ - galosn; rôdn (râtan) - raeat - garôdn; ebenso brôdn (brâtan).

e) $r o^{2} f n$ (hruofan) - $r u^{2} f-g a r o^{2} f n$.

II. Tempusbildung der schwachen verba.

$\S 143$. Die schwachen verba zerfallen im dialekt in zwei klassen. Die erste umfasst einen teil der langsilbigen historischen -ja-verba und bildet das praeteritum mit 'ritckumlaut'; die zweite klasse enthält die tubrige masse schwacher verba.

Das praeteritum beider klassen wird, abgesehen vom 'rluckumlaut' der ersten, durch anhängen eines - $d$ - gebildet, welches den allgemeinen regeln uber die behandlung des ausund anlauts in der composition ( $§ 7)$ unterliegt, da der mittelrocal uberall geschwunden ist.

§ 144. Von starken verbis abgeleitete schwache, welche eine gegensătzlich causative bedeutung bewahrt haben: $l e^{2}$ nan vgl. § 138 a. 1, zoñan (mhd. sengen) und drońan (drengen) vgl.

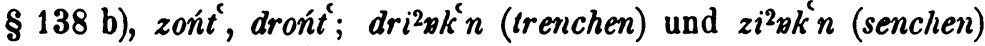
vgl. § $138 \mathrm{~d}) d r i^{2} x t, z i^{2} x t, \nabla g l . \$ 100$ a. 1 ; li'ajn (legen) vgl.

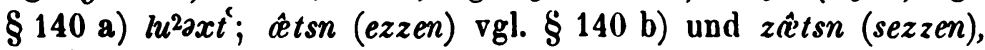
êtst und zatst ${ }^{c}$; frêtsn (*frezzen), frêtst und šmêltsn (smel$z e n$ ), šmêltst sind ableitungen von frôts (frâz) und šmoºlts (smalz).

Anm. raitsn (rizzen) hat nur transitive bedeutung, während $r^{2} e^{2}$ esn (vgl. $\$ 136 \mathrm{~b}$ ) mit dieser auch die intransit. vereinigt. šloe $2 t s n$, einen schlitz machen, zu slizzan a. a. o. ṡmat ${ }^{2} t s n$, zu smizzan, von der schmeissfliege gebraucht; foršma ${ }^{2}$ tst dagegen gleich nhd. verschmitzt und wahrscheinlich nach diesem gebildet. Ueber $r e^{2} n \partial n \mathrm{vgl}$. $\$ 138$ a. 1.

$\S 145$. Verba mit rluckumlaut:

ahd. bougen : bĩjn - bûxt - conj. bî $\chi(t)$

as. brengian : bronən - bru $^{2} \theta x t^{t}-b r \hat{x} \chi t^{t}$

mhd. bticken : $b x^{2} k^{t} n-b^{2} k^{t} t^{t}-b x^{2} k^{t}\left(t^{t}\right)$

ahd. denchen : $d i^{2} m k^{2} n-d u^{2} \theta x t-d \hat{x} x t^{t}$

"dunchan : $d^{2} x k^{k} n-d u^{2} x t^{t}$ und $d u^{2} \partial x t^{t}-d \hat{x} x t^{t}$

"fuolen : fồln - fæalt $-\mathbf{f} \hat{x} l\left(t^{t}\right)$ 
ahd. fuoren : fæ̂rn - fæar't $-f \hat{x} r(\hat{t})$

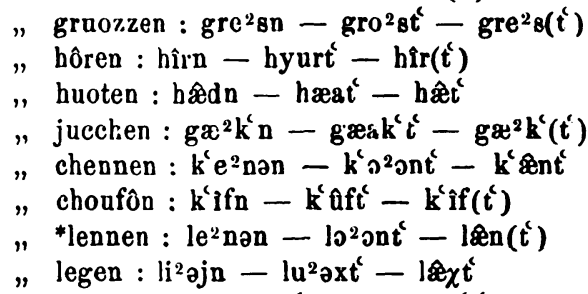

mhd. verrecken : fræ2 $k^{c} n-\operatorname{fr}^{2} a^{2} t^{t}-\operatorname{fr}^{2} k^{c}\left(t^{t}\right)$

ahd. rennen : $\mathrm{re}^{2}$ nən $-\mathrm{ro}^{2} \mathrm{ont}-\mathrm{r} \hat{\mathrm{e} n t}\left(\mathrm{t}^{\mathrm{t}}\right)$

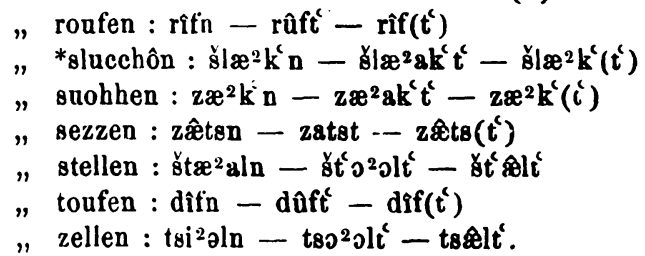

A nm. Mit analogischem 'rückumlant' bilden das praeteritum:

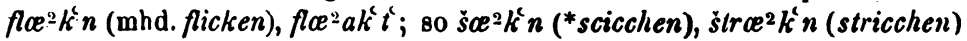
nach analogie von $b a^{2} k^{i} n$ (mhd. bücken) n. ähnl. ḱtirn (ahd. chèrren) hat ähnlich mhd. kêren, kârte praet. k'yurt'.

\section{Tempusbildung der praeteritopraesentia.}

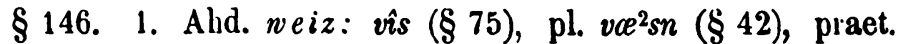
vast (§53), conj. vae $e^{2} t^{t}$, part. gavast, inf. vae $s n$.

2. Abd. kan: k02on $\left(\S 29,1\right.$ a.), k $e^{2} n a n$ (nach analogie

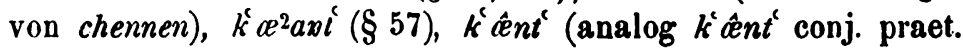
von chennen), gak $a^{2} a x t, k^{k} e^{2} n a n$.

3. Ahd. darf: $t^{2} e^{2} r f, t^{2} e^{2} r f n$ (\$ 54), turft, $t^{i} i^{2} r f t^{\prime}, g^{2}-$ $t u^{2} r f t, t e^{2} r f n$.

4. Ahd. scal: $z 0^{2} \mathrm{Jl}$ ( $\mathrm{vgl}$. dagegen $\S 29$ und $\$ 33,5$ ), $z e^{2} l n$ $(\S 51 \mathrm{~b}), z \hat{a l}$ (§50 und $\S 56), z \hat{\imath} l, z e^{2} l n$.

5. Ahd. muoz: $m e^{2} s, m e^{2} s n(§ 68), m o^{2} s t(\S 68), m e^{2} s t$, ga$m o^{2} s t, m e^{2} s n$.

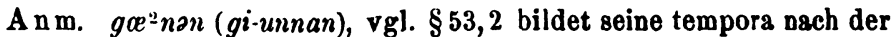
III. ablautsreihe $\$ 138$ a). - Von praeteritopraesentia abgeleitete adjectiva sind $\hat{i} j n$ (eigan) und gat îršs $i^{2} \chi$ (turstigg).

\section{Die personalendungen.}

$\S 147$. Die personalendungen sind bei allen verbis, mit ausnahme de: praetoritopraesentia und einiger nachher năher 
zu bezeichnender 'unregelmåssigen' dieselben. Die endungen des praeteritums werden bei den starken verbis an den praeteritalstamm, bei den schwachen an den um ein $-d$ - resp. $-t$ erweiterten praesensstamm angehängt.

$\S 148$. Die endungen.

Praesens.

Indicativ (conjunctiv).

Sing. 1. -ən, -n (\$11), -ə (\$ 9)

2. $-8 t^{2}$

3. $-t^{c}$

Plur. 1. -on etc.

2. $-t^{t}$

3. .ən etc.

Imperativ.

Sing. 2.

Plur. 2. $-t^{c}$

Infinitiv.

-ən etc. genetiv - $x^{2} n ə s$

Participium.

$-æ^{2} n$

Praeteritum.

Indicativ und conjunctiv.

Sing. 1. -

2. -st.

3. -

Plur. 1. -on otc.

2. $-t^{t}$

3. -ən etc.

A nm. 1. Das -st der II. pers. fällt bei stämmen auf -st mit diesem zusammen: fastn (fasten), 1I. p. sing. ind. praes. und praet.: fast'. Was die ubrigen veränderungen dieser endnng anbelangt vgl. das $§ 121$ a. 2 bezüglich der superlativendung gesagte.

\section{Paradigma: trëtan und bëtôn.}

Praes. ind. sing. 1. tri² ${ }^{2} d n$ bi² $2 d n$

2. tri $^{2}$ tot $^{t} \quad b i^{2} \theta$ tst

3. $\operatorname{tri}^{2} t^{6} \quad b i^{2} \partial t^{t}$

plur. 1. 3. $\operatorname{tri}^{2} \theta d n \quad b i^{2} \partial d n$

2. $t \operatorname{tr}^{2} \theta t^{t} \quad b i^{2} \partial t^{t}$

Imperat. sing. $\operatorname{tri}^{2} t^{t} ! b i^{2} \partial t !-$ plur. tri ${ }^{2} \partial t^{t} ! b i^{2} \partial t !$

Partic. tri ${ }^{2} \partial d \circledast^{2} n, b i^{2} \partial d æ^{2} n$

Infin. tri ${ }^{2} \partial d n, b i^{2} \partial d n-$ genet. $t^{t}$ ri $^{2} \partial d \Re^{2} n o s, b i^{2} \partial d æ^{2} n o s$ 


\begin{tabular}{|c|c|}
\hline $\begin{array}{r}\text { Praet. ind. sing. 1. } 3 . \\
2 . \\
\text { plur. 1. } 3 . \\
2 . \\
\text { conj. sing. 1. } 3 . \\
2 . \\
\text { plur. 1. } 3 . \\
2 . \\
\text { participium }\end{array}$ & 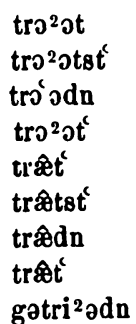 \\
\hline
\end{tabular}

A n m. 2. Im conj. praet. der schwachen verba - er wird meist lurch umschreibungen ersetzt - fällt das praeterit. -t- in der I. III. pers. sing. nach analogie der starken verba hänfig ab. In \$145 habe ich ties durch klammern angedeutet.

Anm. 3. Die flexion einiger starken verba, meist solcher, deren stammauslaut geschwunden, weicht im praesens von der flexion der ibrigen verba ihrer klassen durch die veränderungen $a b$, welche der stammvocal erlitten hat.

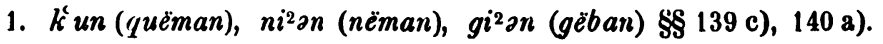

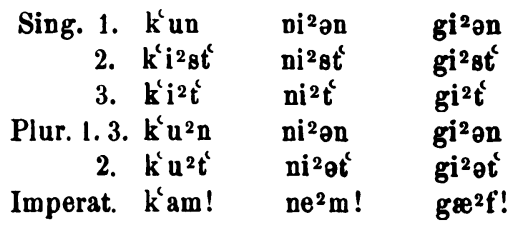

2. šlòn (slahan) und drôn (tragan), vgl. §141, gehen wie gôn und it' on $\$ 149 \mathrm{c})$.

3. zển (sëhan) und gašçen (giscëhan), vgl. §140a):

\begin{tabular}{|c|c|c|}
\hline Sing. & & \\
\hline & & \\
\hline 2. & $\mathrm{ze}^{2} \theta \mathrm{st}$ & - \\
\hline lur. 1.3 & zên & gošæ̂n \\
\hline 2. & zÂt't & 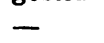 \\
\hline
\end{tabular}

4. $v e^{2} r d n$ (wërdan) vgl. $\left.\$ 138 \mathrm{f}\right)$.

Praes. sing. 1. vææ2 $\Re^{2} d n$

2. vi ${ }^{2}$ rs̀t

3. $v^{2} t^{6}$
Plur. 1. 3. $\quad \nabla æ^{2} \mathbf{r d n}$

2. $\nabla \Re^{2} \mathrm{rt}^{\mathrm{t}}$

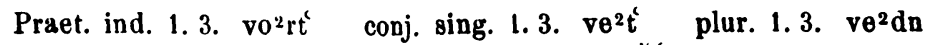
2. vo ${ }^{2} \mathrm{rs̀t}^{\mathrm{s}}$
2. ve ${ }^{2}$ r št $^{\mathrm{s}}$
2. $v e^{2} t$

plur. 1. vo ${ }^{2}$ rdn etc.

5. $0^{2} s n$ (lâzzan) vgl. $\S 142 \mathrm{~d}$.
Sing. 1. $10^{2} \mathrm{sn}$
Plur. 1. $10^{2} \mathrm{gn}$.
2. le ${ }^{2} s t^{t}$
2. $10^{2} 8 t^{6}, 10^{2} t^{t}$
3. $\mathrm{le}^{2} \mathrm{t}$
3. $10^{2} \mathrm{sn}$. 
6. Hierher zu setzen ist auch das schwache $h u^{2} n$ (habin).

Praes. sing. 1. $\mathrm{hu}^{2} \mathrm{n}$

2. hu ${ }^{2}$ est

3. $h u^{2} \partial t^{t}$

plur. 1. 3. $h u^{2} n$

2. $h u^{2} \partial t^{t}$

Imperat. haf!

Praet. ind. sing. 1. 3. hat

2. hast

plur. 1. 3. hadn

2. hat conj. sing. 1. 3. hæ̂t, hæ̂f

2. hâst', hâfst

plur. 1. 3. hæ̂̀n, hæ̂vn

2. hôxt, hôft'

Partic. gohn 2 ot.

$h \hat{c} f$, eigentlich conj. praes., wird wit hêt' promiscue gebraucht. Scine formen werden aber äusserst selten mehr angewendet.

§ 149. Die praeteritopraesentia und die 'unregelmässigen'.

a) Praeteritopraesentia. Es komnt bloss die II. pers. sing. praes. in betracht, welche $[v \hat{i s}], 2 v^{2} s l,\left[m c^{2} s\right]$ mit $t$, [vis $t$,

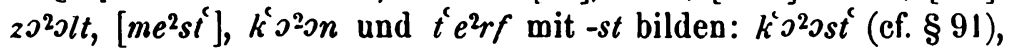
$t^{2} e^{2} r f s t^{2}$.

b) Ahd. wëllan: vaéaln.

Praes. ind. sing. 1. 3. væáal

2. væalt

Prate ind. sing. 1. 3. vâl

2. vûlt

plur. 1. 3. vôln

2. $\nabla \mathbf{a} l t^{t}$ plur. 1. 3. $\quad \mathfrak{x}^{2}$ aln

2. væ2alt

conj. sing. 1. 3. vîl

2. vîlt

1. 3. vîln

2. vîlt

c) Ahd. tuon, gân, stân : dwan, gôn, štôn (vgl. § 141, \$ $142 \mathrm{c})$ :

Praes. ind. sing. 1. dæan

2. dîst

3. dit

plur. 1. 3. dæan

2. dæat

imperat. sing. dæa!

plur. dæat

partic. dæaæ ${ }^{2} \mathbf{n}$ gôn štôn

gîst 'st'ist'

git st It

gôn štón

gôt $t^{\mathfrak{s}} \quad$ št ôt

$\mathrm{go}^{2} \boldsymbol{x k}$ ! št o ont!

gôt! št

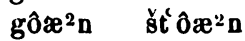

Praet. ind. conj. sing. 1. 3. dêt'; $\operatorname{ge}^{2} x, \mathrm{fe}^{2} x$ regelmässig

2. dêst

plur. 1. 3. dêdn

2. dêt

partic. gədôn. 
d) Ahd. sin, wësan:

Praes. ind. sing. 1. bæ2n plur. 1. ze $e^{2} n$ und zen (vgl. §9)
2. $b x^{2} s t^{t}$
2. $z e^{2} t^{t}$ und $z e t^{\prime} t$

3. $\mathfrak{x}^{2} \mathbf{s}$

3. ze ${ }^{2} n$ und zeń

imperat. $\mathrm{zo}^{2} \mathrm{o}^{2} ! \mathrm{zo}^{2} \mathrm{o}^{2} t$ ! infin. $\mathrm{ze}^{2} \mathrm{n}$ und zeń

Praet. ind. sing. 1. 3. vôr

2. vôršt

plur. 1. 3. vôrn

2. vôrt' conj. sing. 1. 3. vêr

2. vêršt

plur. 1. 3. vêrn

2. vêrt't

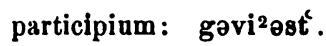

MARK'TSCHELKEN, märz 1886.

ANDREAS SCHEINER. 